See discussions, stats, and author profiles for this publication at: https://www.researchgate.net/publication/336409271

\title{
On the application of isogeometric finite volume method in numerical analysis of wet-steam flow through turbine cascades
}

Article in Computers \& Mathematics with Applications · October 2019

DOI: 10.1016/j.camwa.2019.09.025

CITATIONS

READS

0

28

2 authors:

A. Ali Hashemian

Basque Center for Applied Mathematics

15 PUBLICATIONS 82 CITATIONS

SEE PROFILE

2. Esmail Lakzian

Hakim Sabzevari University

89 PUBLICATIONS 169 CITATIONS

SEE PROFILE

Some of the authors of this publication are also working on these related projects:

Recent Achivements in Computer-aided Design View project

Project Tolerance Analysis of Flexible Sheet Metal Structures View project 


\title{
On the Application of Isogeometric Finite Volume Method in Numerical Analysis of Wet-Steam Flow through Turbine Cascades
}

\author{
Ali Hashemian ${ }^{1,2}$, Esmail Lakzian ${ }^{3 *}$, Amir Ebrahimi-Fizik ${ }^{3}$ \\ 1. BCAM - Basque Center for Applied Mathematics, Bilbao, Basque Country, Spain \\ 2. Department of Mechanical Engineering, Hakim Sabzevari University, Sabzevar, Iran \\ 3. Center of Computational Energy, Department of Mechanical Engineering, Hakim Sabzevari University, \\ Sabzevar, Iran \\ * Corresponding author, email address: e.lakzian@ @su.ac.ir, Tel/Fax.: +98 5144012818
}

\begin{abstract}
The isogeometric finite volume analysis is utilized in this research to numerically simulate the twodimensional viscous wet-steam flow between stationary cascades of a steam turbine for the first time. In this approach, the analysis-suitable computational mesh with "curved" boundaries is generated for the fluid flow by employing a non-uniform rational B-spline (NURBS) surface that describes the cascade geometry, and the governing equations are then discretized by the NURBS representation. Thanks to smooth and accurate geometry representation of the NURBS formulation, the employed isogeometric framework not only resolves issues concerning the conventional mesh generation techniques of the finite volume method in steam turbine problems, but also, as validated against well-established experiments, significantly improves the accuracy of the numerical solution. In addition, the shock location in the cascade is predicted and tracked with a sufficient accuracy.
\end{abstract}

Keywords: wet-steam flow; turbine cascades; NURBS surface; isogeometric analysis; finite volume method

\section{Introduction}

The concept of isogeometric analysis (IGA), first introduced by Hughes et al. [1], tries to significantly bridge the gap between computer-aided design (CAD) and computational physics/mechanics by employing the same representation for both the geometry and solution. More precisely, in the isogeometric framework, the computational mesh is inherently created by non-uniform rational B-spline (NURBS) description of the geometry and the governing equations are then discretized by transforming from the "curved" grids (of arbitrary degree) on the computational domain to the "rectangular" ones on the parameter space of the Bspline/NURBS geometry. Thanks to the accurate and efficient geometry representation of the IGA approach, it has been successfully implemented in various practical applications such as computational mathematics [2-5], electromagnetics [6, 7], solid and fluid mechanics [8-12], fluid-structure interaction $[13,14]$, heat transfer $[15,16]$ and eigenvalue problems $[17,18]$.

Postprint accepted by Elsevier journal: Computers \& Mathematics with Applications, published online on doi.org/10.1016/j.camwa.2019.09.025 
A common problem in the last stages of low-pressure (LP) steam turbines is "condensation shock" that can be interpreted as a non-equilibrium and unsteady thermodynamic phenomenon. Due to the negative effects of condensation on the performance of steam turbines, this phenomenon has been investigated in many studies both numerically [19-23] and experimentally [24-29]. During the expansion of steam, under certain circumstances in LP steam turbines, the one-phase vapor changed into a two-phase mixture after supercooling and nucleation. The liquid phase of the mixture causes mechanical and thermodynamic losses and, as a result, a reduction in the efficiency of the turbines. In order to improve the modeling accuracy and enhance the physical understanding of such "wet-steam flow" in turbine cascades, majority of studies with the aim of correcting the nucleation equation, adopting more appropriate droplet growth model, and considering the slip condition effects and coalescence of droplets have been performed (see, e.g., [30-34]). Furthermore, the accuracy of the wet-steam flow simulation in turbine cascades, like other problems in computational fluid dynamics (CFD), significantly depends on the accuracy and quality of the computational mesh. Some common mesh types that can be found in the literature are, e.g., O-mesh [35, 36], quasi grid [37, 38], and H-type grid [39-41]. On the other hand, when it comes to finite volume method (FVM), as one of the most frequent numerical approaches in CFD, it is of interest to discretize the computational domain by volumes (or cells) with curved boundaries that is, indeed, a common bottleneck in most of common grid generation techniques. The mentioned difficultly, however, can be considerably resolved by employing the isogeometric finite volume analysis.

In this paper, by employing the isogeometric finite volume analysis (presented by Heinrich et al. [42]) the numerical modeling of the two-phase viscous flow in turbine cascades is performed for the first time using the isogeometric framework. Given the smooth and accurate geometry representation of the NURBS formulation, the main advantage of the employed methodology is in accounting for curved control volumes in the FVM approach that is well consistent with the geometry of the cascades and enhances the accuracy of results. For this purpose, the Eulerian-Eulerian model combined with the $k-\omega$ turbulence formulation is used to analyze the viscous wet-steam flow in the last stages of an LP steam turbine. Additionally, the corrected Courtney-Kantrowitz nucleation model [43, 44], and the Young droplet growth model [45] are employed to simulate the condensation phenomenon. The weak forms of the governing equations are then created using discontinuous Galerkin method and discretized by NURBS representation. The results, validated by well-established experiments, indicate that employing IGA approach will significantly improve the numerical solution outputs as compared to the cases in which a conventional mesh (e.g., $\mathrm{H}$ type grid) is used.

The remainder of this paper is organized as follows. Section 2 presents the governing equations of the wetsteam flow. In Section 3, the isogeometric framework integrated with finite volume analysis is introduced. Section 4 expresses the modeling process of wet-steam flow in turbine cascades. The grid generation as well as results validation is also studied in this section, followed by Section 5 that presents the numerical simulation results. Finally, Section 6 draws some conclusion. 


\section{Governing equations of wet-steam flow}

In this section, we present the governing wet-steam flow formulation.

\subsection{Conservation equations}

The conservation equations are the Reynolds-averaged time dependent compressible Navier-Stokes equations that can be written for a two-dimensional flow as follows (see, e.g., [46, 47]):

$$
\begin{aligned}
& \frac{\partial \rho}{\partial t}+\nabla \cdot(\rho \boldsymbol{u})=0 \\
& \frac{\partial(\rho \boldsymbol{u})}{\partial t}+\boldsymbol{\nabla} \cdot(\rho \boldsymbol{u} \otimes \boldsymbol{u})+\nabla P-\nabla \cdot \boldsymbol{\tau}=0 \\
& \frac{\partial e}{\partial t}+\boldsymbol{\nabla} \cdot[(e+P) \boldsymbol{u}]-\boldsymbol{\nabla} \cdot(\boldsymbol{\tau} \cdot \boldsymbol{u})-\boldsymbol{\nabla} \cdot \boldsymbol{q}=0
\end{aligned}
$$

where the energy term $e$, viscous stress tensor $\boldsymbol{\tau}$ and heat flux $\boldsymbol{q}$ are given by:

$$
\begin{aligned}
& e=\frac{P}{\gamma-1}+\frac{1}{2} \rho \boldsymbol{u} \cdot \boldsymbol{u} \\
& \boldsymbol{\tau}=\mu\left[(\boldsymbol{\nabla} \boldsymbol{u})^{T}+\boldsymbol{\nabla} \boldsymbol{u}\right]-\frac{2}{3} \mu(\boldsymbol{\nabla} \cdot \boldsymbol{u}) \boldsymbol{I} \\
& \boldsymbol{q}=K \boldsymbol{\nabla} T
\end{aligned}
$$

In the above equations, $\boldsymbol{\nabla}=\left[\frac{\partial}{\partial x} \frac{\partial}{\partial y}\right]^{T}$ is the gradient vector, $\boldsymbol{I}$ is the identity matrix, $\boldsymbol{u}=\left[\begin{array}{ll}u & v\end{array}\right]^{T}$ is the velocity vector in the $x-y$ coordinate system and $\gamma$ is the specific heat ratio. In addition, $P, T, K$, and $\mu$ correspond to pressure, temperature, effective thermal conductivity, and viscosity, respectively. Finally, $\rho$ is the density of the mixture that is related to the density of the vapor phase $\rho_{v}$ and mass fraction of the liquid phase $\beta$ as follows:

$$
\rho=\frac{\rho_{v}}{(1-\beta)}
$$

It is important to note that the conservation equations are expressed with regard to the properties of the two-phase mixture which can be estimated by the respective properties of the liquid and vapor phases as [48]:

$$
\varphi=\varphi_{l} \beta+(1-\beta) \varphi_{v}
$$

where $\varphi$ refers to the mixture properties (e.g., enthalpy, entropy, specific heat, viscosity, and thermal conductivity) and subscripts $l$ and $v$ refer to the liquid and vapor phases, respectively.

In order to model the wet-steam condensation flow, two additional transport equations should be taken into consideration as follows: 


$$
\begin{aligned}
& \beta \frac{\partial \rho}{\partial t}+\boldsymbol{\nabla} \cdot(\beta \rho \boldsymbol{u})=\Gamma \\
& \eta \frac{\partial \rho}{\partial t}+\nabla \cdot(\eta \rho \boldsymbol{u})=\rho I
\end{aligned}
$$

The first equation is related to the mass fraction of the liquid phase $\beta$ where $\Gamma$ is defined as the mass generation rate per unit volume per second and is due to condensation and evaporation. The second transport equation estimates the number of liquid droplet per unit volume (designated as $\eta$ ) where $I$ shows the nucleation rate in number of generated droplets per unit volume per second. The computation procedures of $I$ and $\Gamma$ will be discussed in the following subsection. Finally, the number of droplets $\eta$ is related to the mass fraction $\beta$ by the following equation [49]:

$$
\eta=\frac{\beta}{(1-\beta) V_{d}\left(\rho_{l} / \rho_{v}\right)}
$$

where $V_{d}=\frac{4}{3} \pi \bar{r}_{d}^{3}$ denotes the average droplet volume obtained by the average droplet radius $\bar{r}_{d}$.

\section{$2.2 \quad$ Nucleation and droplet growth models}

The condensation phenomenon consisting two main processes of nucleation and droplet growth. The classical nucleation theory presents the nucleation and formation of spherical droplets from a supersaturated condition. Hence, a steady-state homogenous nucleation rate is formulated as follows [41]:

$$
I_{\text {classic }}=q_{c} \frac{\rho_{v}^{2}}{\rho_{l}} \sqrt{\frac{2 \sigma}{\pi M_{m}^{3}}} \exp \left(-\frac{4 \pi r^{* 2} \sigma}{3 k_{b} T_{v}}\right)
$$

In the above equation, $q_{c}, k_{b}, M_{m}$, and $\sigma$ are, respectively, the condensation coefficient, Boltzmann constant, molecular mass, and liquid surface tension and $r^{*}$ is the Kelvin-Helmholtz critical droplet radius given by [50]:

$$
r^{*}=\frac{2 \sigma}{\rho_{l} R T \ln S_{r}}
$$

where $R$ is the gas constant and $S_{r}$ is the supersaturation ratio defined as the ratio of the vapor pressure to the equilibrium saturation pressure [20]. Since the expansion of vapor is occurred rapidly, the process changes into non-equilibrium condition. As a result, the supersaturation ratio can be greater than one [41]. A variety of corrections is proposed to apply on the classic nucleation theory for non-isothermal homogeneous condensation, where the accuracy of the Courtney-Kantrowitz correction approach has been proven by previous studies (see, e.g., $[38,51,52]$ ). Hence, the nucleation rate is to be computed as follows where that $\theta$ denotes the non-isothermal correction and $h_{l v}$ is the enthalpy of evaporation [53]: 


$$
\begin{aligned}
& I=\frac{1}{(1+\theta)} I_{\text {classic }} \\
& \theta=2 \frac{\gamma-1}{\gamma+1}\left(\frac{h_{l v}}{R T}\right)\left(\frac{h_{l v}}{R T}-\frac{1}{2}\right)
\end{aligned}
$$

In the classical nucleation theory, the rate of mass generation is defined as the total mass increase by the nucleation and growth of droplets as follows [49]:

$$
\Gamma=\frac{4}{3} \pi \rho_{l} I r^{* 3}+4 \pi \rho_{l} \eta \bar{r}^{2} \frac{\partial \bar{r}}{\partial t}
$$

In the above equation, $\partial \bar{r} / \partial t$ is the droplet growth rate obtained by the following equation where $C_{P}$ is specific heat of liquid [54]:

$$
\frac{\partial \bar{r}}{\partial t}=\frac{P}{h_{l v} \rho_{l} \sqrt{2 \pi R T}} \frac{\gamma+1}{2 \gamma} C_{P}\left(T_{l}-T_{v}\right)
$$

Hence, for determining the droplet temperature $T_{l}$ as a function of vapor temperature $T_{v}$, supercooling degree $T_{s}$ and droplet radius $r$, Eq. (12) should be employed where the droplet radius is assumed to be $1 \mu \mathrm{m}$ or less [55]:

$$
T_{l}=T_{S}(P)-\left[T_{S}(P)-T_{v}\right] \frac{r^{*}}{r}
$$

\subsection{Equation of state}

The vapor's pressure, temperature and density are related by means the equation of state as follows [56]:

$$
P=\rho_{v} R T\left(1+B \rho_{v}+C \rho_{v}^{2}\right)
$$

where $B$, and $C$ are the second and third virial coefficients given in $\mathrm{m}^{3} / \mathrm{kg}$ and $\mathrm{m}^{6} / \mathrm{kg}^{2}$, respectively and defined as follows:

$$
\begin{aligned}
& B=b_{1}\left(1+\frac{\tau_{B}}{\alpha_{B}}\right)^{-1}+b_{2} e^{\tau_{B}}\left(1-e^{-\tau_{B}}\right)^{5 / 2}+b_{3} \tau_{B} \\
& C=c_{1}\left(\tau_{C}-\tau_{0}\right) e^{-\alpha_{C} \tau_{C}}+c_{2}
\end{aligned}
$$

The parameters involved in the above equations are expressed in Table 1.

Table 1. Parameters of the equation of state

\begin{tabular}{cccccccccc}
\hline$\tau_{B}$ & $\tau_{C}$ & $\tau_{0}$ & $\alpha_{B}$ & $\alpha_{C}$ & $b_{1}$ & $b_{2}$ & $b_{3}$ & $c_{1}$ & $c_{2}$ \\
\hline $1500 / T$ & $T / 647.286$ & 0.89780 & 10000 & 11.16 & 0.0015 & 0.000942 & -0.0004882 & 1.722 & $1.5 \times 10^{-6}$ \\
\hline
\end{tabular}




\section{$2.4 \quad$ Turbulence model}

In the present paper, the $k-\omega$ turbulence model is employed to simulate the turbulent wet-steam flow through turbine cascades. This common turbulence model accounts for wall effects and attempts to predict turbulence by two partial differential equations as follows $[57,58]$. The equations are in terms of two variables $k$ and $\omega$, which are the turbulence kinetic energy and specific rate of dissipation, respectively, noting that the coefficients of the equations are $\alpha_{0}=5 / 9, \beta_{0}=3 / 40, \beta^{*}=9 / 100$, and $\sigma_{0}=\sigma^{*}=1 / 2$.

$$
\begin{aligned}
& \frac{\partial(\rho k)}{\partial t}+\nabla \cdot(\rho k \boldsymbol{u})=\boldsymbol{\tau} \cdot \boldsymbol{\nabla u}-\beta^{*} \rho k \omega+\boldsymbol{\nabla} \cdot\left[\left(\mu+\sigma^{*} \frac{\rho k}{\omega}\right) \boldsymbol{\nabla} k\right] \\
& \frac{\partial(\rho \omega)}{\partial t}+\boldsymbol{\nabla} \cdot(\rho \omega \boldsymbol{u})=\alpha_{0} \frac{\omega}{k} \boldsymbol{\tau} \cdot \nabla \boldsymbol{u}-\beta_{0} \rho \omega^{2}+\nabla \cdot\left[\left(\mu+\sigma_{0} \frac{\rho k}{\omega}\right) \boldsymbol{\nabla} \omega\right]
\end{aligned}
$$

\section{Isogeometric finite volume analysis}

\subsection{A brief review of B-spline surfaces}

NURBS formulation is considered as a universal class for geometry modeling in computer-aided design, manufacture and engineering (CAD/CAM/CAE). The trajectory of a robot [59], geometry of a curved structure [60-63], free surface of a fluid [64], a tool-path in multi-axis machining [65, 66] and automotive body panels [67-69] are common examples described by NURBS formulation.

B-splines, as unweighted or non-rational form of NURBS surfaces, can model a rich variety of free-form geometries and are well consistent with commercial CAD software with different engineering applications. Along with other geometry representation methods of the IGA approach (like, e.g., elliptic [70] and Tspline [71] formulations), B-splines play a central role in the isogeometric finite volume analysis of flows thorough turbine cascades since they serve both for the geometric representation of the physical domain and for discretization of the governing equations. In the present paper, the physical domain of the fluid flow between turbine cascades can be interpreted by a planar B-spline surface as a mapping from the bivariate parameter space $(\xi, \zeta) \in[0,1]^{2}$ onto the geometric (i.e. physical) space $\boldsymbol{S}(\xi, \zeta)=[x(\xi, \zeta), y(\xi, \zeta)]$. This B-spline surface of degree $(p, q)$ is characterized by a bidirectional net of $(n+1)(m+1)$ control points $\boldsymbol{P}_{i j}=\left[P_{x}^{i j}, P_{y}^{i j}\right]$ and defined as:

$$
\boldsymbol{S}(\xi, \zeta)=\sum_{j=0}^{m} \sum_{i=0}^{n} N_{i, p}(\xi) N_{j, q}(\zeta) \boldsymbol{P}_{i j}
$$

where $N_{i, p}(\xi)$ and $N_{j, q}(\zeta)$ are the $i$-th and $j$-th basis functions of degree $p$ and $q$, respectively, and defined by the Cox-de Boor recursion formula over the non-decreasing knot vectors $\boldsymbol{\Xi}$ and $\boldsymbol{Z}$ as follows [72].

$$
\begin{aligned}
& \boldsymbol{\Xi}=[\underbrace{0,0, \cdots, 0}_{p+1}, \xi_{p+1}, \xi_{p+2}, \cdots, \xi_{n}, \underbrace{1,1, \cdots, 1}_{p+1}] \\
& \boldsymbol{Z}=[\underbrace{0,0, \cdots, 0}_{q+1}, \zeta_{q+1}, \zeta_{q+2}, \cdots, \zeta_{m}, \underbrace{1,1, \cdots, 1}_{q+1}]
\end{aligned}
$$




$$
\begin{aligned}
& N_{i, 0}(\xi)= \begin{cases}1 & \xi_{i} \leq \xi<\xi_{i+1} \\
0 & \text { otherwise }\end{cases} \\
& N_{i, p}(\xi)=\frac{\xi-\xi_{i}}{\xi_{i+p}-\xi_{i}} N_{i, p-1}(\xi)+\frac{\xi_{i+p+1}-\xi}{\xi_{i+p+1}-\xi_{i+1}} N_{i+1, p-1}(\xi)
\end{aligned}
$$

According to the tensor-product characteristic of the NURBS format, for a B-spline surface of degree $(p, q)$ with a $(n+1)(m+1)$ control net, the product of the knot vectors $\boldsymbol{\Xi}$ and $\boldsymbol{Z}$ results in a set of $(n-p+1)(m-q+1)$ patches on the surface that correspond to non-zero knot spans of the knot vectors. In the isogeometric finite volume analysis, every "curved" patch $\Omega^{k}$ on the geometric domain, as the map of the respective $\Omega_{0}^{k}$ of the parameter space, is considered as a control volume in the physical space. As illustrated for a bicubic (i.e., $p=q=3$ ) surface in Fig. 1, the local support property of B-spline definition necessitates that each patch has $p+1$ and $q+1$ non-zero (i.e., active) basis functions in $\xi$ and $\zeta$ directions, respectively. This property will then be helpful in isogeometric discretization of the computational domain, since only the active basis functions will participate in the numerical integration. As stated earlier, the advantage of incorporating IGA and FVM approaches lies in accounting for control volumes with curved boundaries that enhance the accuracy of results.

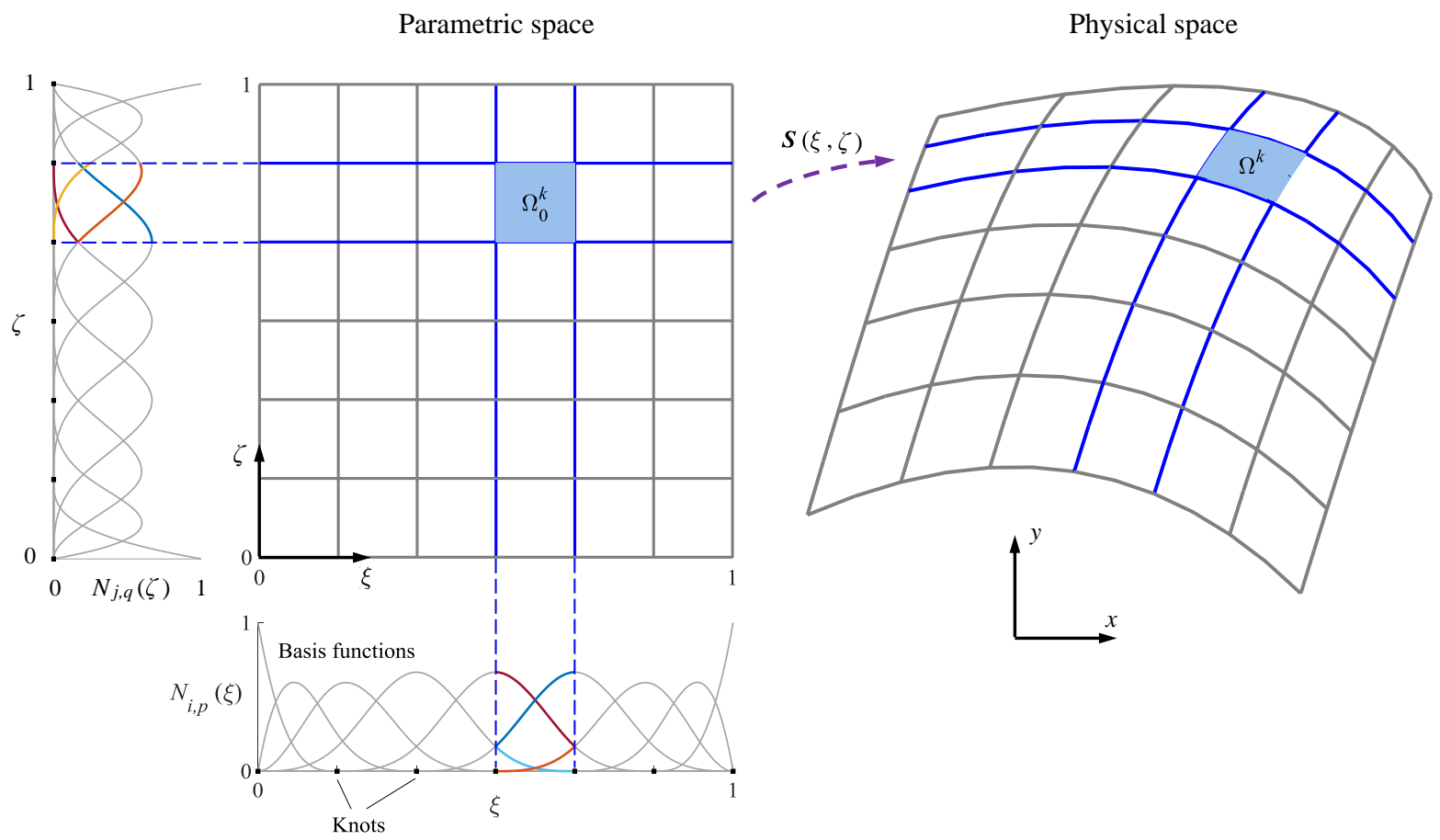

Fig. 1. A bicubic B-spline surface in the physical space (right) and its corresponding domain in the parameter space with respective basis functions (left) [73]

\subsection{Weak formulation}

By partitioning the physical domain into finite volumes $\Omega^{k}, k=1,2, \cdots,(n-p+1)(m-q+1)$, the strong forms of governing equations, i.e. Eqs. (1), (5) and (15), are expressed in the weak forms as follows based on the standard discontinuous Galerkin (DG) method described in, e.g., [42, 74]. 


$$
\begin{aligned}
& \int_{\Omega^{k}} \frac{\partial \rho}{\partial t} d \Omega+\int_{S^{k}}(\hat{\rho} \widehat{\boldsymbol{u}}) \cdot \boldsymbol{n} d s=0 \\
& \int_{\Omega^{k}} \frac{\partial(\rho \boldsymbol{u})}{\partial t} d \Omega+\int_{s^{k}}(\hat{\rho} \widehat{\boldsymbol{u}} \otimes \widehat{\boldsymbol{u}}) \cdot \boldsymbol{n} d s+\int_{S^{k}} \hat{\boldsymbol{P}} \boldsymbol{n} d s-\mu \int_{S^{k}}\left\{\left[(\boldsymbol{\nabla} \widehat{\boldsymbol{u}})^{T}+\boldsymbol{\nabla} \widehat{\boldsymbol{u}}\right]-\frac{2}{3}(\boldsymbol{\nabla} \cdot \widehat{\boldsymbol{u}}) \boldsymbol{I}\right\} \cdot \boldsymbol{n} d s=0 \\
& \int_{\Omega^{k}} \frac{\partial}{\partial t}\left(\frac{P}{\gamma-1}+\frac{1}{2} \rho \boldsymbol{u} \cdot \boldsymbol{u}\right) d \Omega+\int_{S^{k}}\left(\frac{\hat{P}}{\gamma-1}+\frac{1}{2} \hat{\rho} \widehat{\boldsymbol{u}} \cdot \widehat{\boldsymbol{u}}+\hat{P}\right) \widehat{\boldsymbol{u}} \cdot \boldsymbol{n} d s \\
& -\mu \int_{s^{k}}\left(\left\{\left[(\boldsymbol{\nabla} \widehat{\boldsymbol{u}})^{T}+\boldsymbol{\nabla} \widehat{\boldsymbol{u}}\right]-\frac{2}{3}(\boldsymbol{\nabla} \cdot \widehat{\boldsymbol{u}}) \boldsymbol{I}\right\} \cdot \widehat{\boldsymbol{u}}\right) \cdot \boldsymbol{n} d s-K \int_{s^{k}} \boldsymbol{\nabla} \hat{T} \cdot \boldsymbol{n} d s=0 \\
& \int_{\Omega^{k}}\left(\beta \frac{\partial \rho}{\partial t}-\Gamma\right) d \Omega+\int_{s^{k}}(\hat{\beta} \hat{\boldsymbol{\mu}} \widehat{\boldsymbol{u}}) \cdot \boldsymbol{n} d s=0 \\
& \int_{\Omega^{k}}\left(\eta \frac{\partial \rho}{\partial t}-\rho I\right) d \Omega+\int_{S^{k}}(\hat{\eta} \hat{\rho} \widehat{\boldsymbol{u}}) \cdot \boldsymbol{n} d s=0 \\
& \int_{\Omega^{k}}\left[\frac{\partial(\rho k)}{\partial t}+\beta^{*} \rho k \omega\right] d \Omega+\int_{s^{k}}(\hat{\rho} \hat{k} \widehat{\boldsymbol{u}}) \cdot \boldsymbol{n} d s \\
& -\mu \int_{S^{k}}\left(\left\{\left[(\nabla \widehat{\boldsymbol{u}})^{T}+\nabla \widehat{\boldsymbol{u}}\right]-\frac{2}{3}(\boldsymbol{\nabla} \cdot \widehat{\boldsymbol{u}}) \boldsymbol{I}\right\} \cdot \widehat{\boldsymbol{u}}\right) \cdot \boldsymbol{n} d s-\int_{S^{k}}\left[\left(\mu+\sigma^{*} \frac{\hat{\rho} \hat{k}}{\widehat{\omega}}\right) \boldsymbol{\nabla} \hat{k}\right] \cdot \boldsymbol{n} d s=0 \\
& \int_{\Omega^{k}}\left[\frac{\partial(\rho \omega)}{\partial t}+\beta_{0} \rho \omega^{2}\right] d \Omega+\int_{s^{k}}(\hat{\rho} \widehat{\omega} \widehat{\boldsymbol{u}}) \cdot \boldsymbol{n} d s \\
& -\mu \int_{S^{k}}\left(\alpha_{0} \frac{\widehat{\omega}}{\hat{k}}\left\{\left[(\nabla \widehat{\boldsymbol{u}})^{T}+\nabla \widehat{\boldsymbol{u}}\right]-\frac{2}{3}(\boldsymbol{\nabla} \cdot \widehat{\boldsymbol{u}}) \boldsymbol{I}\right\} \cdot \widehat{\boldsymbol{u}}\right) \cdot \boldsymbol{n} d s-\int_{S^{k}}\left[\left(\mu+\sigma_{0} \frac{\hat{\rho} \hat{k}}{\widehat{\omega}}\right) \boldsymbol{\nabla} \widehat{\omega}\right] \cdot \boldsymbol{n} d s=0
\end{aligned}
$$

In the above weak formulation, two different integration schemes are employed. 1) Integration over the control volume $\Omega^{k}$ where the integrands are in terms of field variables within the control volume (i.e., $\phi=[\rho, u, v, P, T, \beta, \eta, k, \omega])$; and 2) boundary integrals over faces $s^{k}$ of the control volumes noting that the integrands are in terms of flux variables passing through the faces (i.e., $\hat{\phi}=[\hat{\rho}, \hat{u}, \hat{v}, \hat{P}, \hat{T}, \hat{\beta}, \hat{\eta}, \hat{k}, \widehat{\omega}]$ ).

\subsection{Isogeometric discretization}

In the isogeometric finite volume analysis, the discretization technique is performed by means of BSpline/NURBS formulation. For this purpose, the underlying integral formulation of the FVM needs to be transformed to the parametric domain that describes the geometry. As a result, control volumes with curved boundaries can be considered in the physical space aiming at improving the accuracy of results. As stated earlier in $\S 3.1$, the patches on the B-spline surface of the physical domain are equivalent to control volumes and, therefore, any additional procedure for generating computational meshes is eliminated and the computational domain is well consistent with the geometry.

In order to compute the integrals of Eq. (19), a transformation from the physical domain $\Omega^{k}$ to the parametric space $\Omega_{0}^{k}$ is required (see Fig. 1). This transformation is performed by means of the Jacobian matrix and its determinant given by: 


$$
\boldsymbol{J}=\left[\begin{array}{ll}
\frac{\partial x}{\partial \xi} & \frac{\partial x}{\partial \zeta} \\
\frac{\partial y}{\partial \xi} & \frac{\partial y}{\partial \zeta}
\end{array}\right] \quad, \quad J=\operatorname{det}(\boldsymbol{J})
$$

where the derivatives are computed based on the B-spline representation of the geometry as [72]:

$$
\begin{aligned}
& {\left[\frac{\partial x}{\partial \xi}, \frac{\partial y}{\partial \xi}\right]=\sum_{j=0}^{m} \sum_{i=0}^{n} \frac{d N_{i, p}(\xi)}{d \xi} N_{j, q}(\zeta) \boldsymbol{P}_{i j}} \\
& {\left[\frac{\partial x}{\partial \zeta}, \frac{\partial y}{\partial \zeta}\right]=\sum_{j=0}^{m} \sum_{i=0}^{n} N_{i, p}(\xi) \frac{d N_{j, q}(\zeta)}{d \zeta} \boldsymbol{P}_{i j}}
\end{aligned}
$$

Hence, the gradient and normal vectors in the Cartesian space $x-y$ can be related to their respective vectors in the parameter space $\xi-\zeta$ as:

$$
\begin{array}{ll}
\boldsymbol{\nabla}=\boldsymbol{J}^{-T} \boldsymbol{\nabla}_{0} \quad, \quad \boldsymbol{\nabla}_{0}=\left[\begin{array}{c}
\frac{\partial}{\partial \xi} \\
\frac{\partial}{\partial \zeta}
\end{array}\right] \\
\boldsymbol{n}=|J| \boldsymbol{J}^{-T} \boldsymbol{n}_{0} \quad, \quad \boldsymbol{n}_{0}=\left[\begin{array}{l}
n_{\xi} \\
n_{\zeta}
\end{array}\right]
\end{array}
$$

For any arbitrary integral over the control volume $\Omega^{k}$, the standard transformation to the parametric space $\Omega_{0}^{k}$ implies that:

$$
\int_{\Omega^{k}} f(x, y) d \Omega=\int_{\Omega_{0}^{k}} f(x(\xi, \zeta), y(\xi, \zeta))|J| d \xi d \zeta
$$

while for the boundary integrals, based on the definition of the inner product $\left(\boldsymbol{a} \cdot \boldsymbol{b}=\boldsymbol{a}^{T} \boldsymbol{b}\right)$, one can obtain for some terms of Eq. (19) that:

$$
\begin{aligned}
& \int_{s^{k}} \boldsymbol{\nabla} \hat{T} \cdot \boldsymbol{n} d s=\int_{s_{0}^{k}}\left(\nabla_{0} \widehat{T}\right)^{T} \boldsymbol{J}^{-1} \boldsymbol{J}^{-T} \boldsymbol{n}_{0}|J| d s_{0} \\
& \int_{s^{k}} \boldsymbol{\nabla} \hat{\boldsymbol{u}} \cdot \boldsymbol{n} d s=\int_{s_{0}^{k}}\left(\nabla_{0} \widehat{\boldsymbol{u}}\right)^{T} \boldsymbol{J}^{-1} \boldsymbol{J}^{-T} \boldsymbol{n}_{0}|J| d s_{0} \\
& \int_{S^{k}} \hat{P} \boldsymbol{n} d s=\int_{s_{0}^{k}} \hat{P} \boldsymbol{J}^{-T} \boldsymbol{n}_{0}|J| d s_{0} \\
& \int_{S^{k}}(\hat{\rho} \widehat{\boldsymbol{u}}) \cdot \boldsymbol{n} d s=\int_{S_{0}^{k}} \hat{\rho} \widehat{\boldsymbol{u}}^{T} \boldsymbol{J}^{-T} \boldsymbol{n}_{0}|J| d s_{0} \\
& \int_{s^{k}}(\hat{\rho} \widehat{\boldsymbol{u}} \otimes \widehat{\boldsymbol{u}}) \cdot \boldsymbol{n} d s=\int_{s_{0}^{k}}(\hat{\rho} \widehat{\boldsymbol{u}} \otimes \widehat{\boldsymbol{u}})^{T} \boldsymbol{J}^{-T} \boldsymbol{n}_{0}|J| d s_{0}
\end{aligned}
$$


Considering that $d s_{0}=d \xi$ (when $d \zeta=0$ ) and $d s_{0}=d \zeta$ (when $d \xi=0$ ), each boundary integral should be split up into four integrals over the northern (n), eastern (e), western (w) and southern (s) faces of the control volume. For instance, the first integral of Eq. (24) is rewritten as:

$$
\int_{S^{k}} \boldsymbol{\nabla} \hat{T} \cdot \boldsymbol{n} d s=\int_{s_{0}^{k}}\left(\boldsymbol{\nabla}_{0} \widehat{T}\right)^{T} \boldsymbol{J}^{-1} \boldsymbol{J}^{-T} \boldsymbol{n}_{0}|\boldsymbol{J}| d s_{0}=\sum_{l=n, e, w, s}\left[\int_{s_{0}^{k, l}}\left(\boldsymbol{\nabla}_{0} \widehat{T}_{l}\right)^{T} \boldsymbol{J}^{-1} \boldsymbol{J}^{-T} \boldsymbol{n}_{0}^{l}|\boldsymbol{J}| d s_{0}\right]
$$

\subsection{Finite volume analysis}

A common procedure in the FVM approach is to presume the field variables to be constant values in each control volume. Hence, considering $\phi$ as the representative of the dependent field variables, it needs to be approximated over the computational domain by its discrete counterparts $\bar{\phi}^{k}$ which are constant in the respective control volumes $\Omega^{k}$. The values of $\bar{\phi}^{k}$ are then to be interpolated by B-spline formulation to find the distributed values of $\phi$ through the cascade. Additionally, by introducing the computational node at the center (P) of the control volume (see Fig. 2), the fluxes and their gradients through faces (n), (e), (w), and (s) can be expressed in terms of the approximated field variables in neighboring control volumes, e.g., (N), $(\mathrm{E}),(\mathrm{W})$ and $(\mathrm{S})$. For this purpose, the upwind difference scheme $[75,76]$ is employed to take into account the effect of flow direction in the analysis. Hence, referring to Fig. 2 and assuming the flow direction is from left to right, the flux through eastern face of the control volume would be $\widehat{\phi}_{e}=\bar{\phi}_{P}+\left(\xi_{e}-\xi_{P}\right) \frac{\partial \widehat{\phi}_{e}}{\partial \xi}$. As a result, by defining the flux gradient as

$$
\frac{\partial \hat{\phi}_{e}}{\partial \xi}=\frac{\bar{\phi}_{E}-\bar{\phi}_{W}}{\xi_{E}-\xi_{W}}
$$

one can write

$$
\widehat{\phi}_{e}=\bar{\phi}_{P}+\alpha\left(\bar{\phi}_{E}-\bar{\phi}_{W}\right)
$$

where $\alpha=\frac{\xi_{e}-\xi_{P}}{\xi_{E}-\xi_{W}}$. The flux gradient with respect to $\zeta$ can also be calculated as:

$$
\frac{\partial \hat{\phi}_{e}}{\partial \zeta}=\frac{\bar{\phi}_{N}-\bar{\phi}_{S}+\alpha\left(\bar{\phi}_{N E}-\bar{\phi}_{S E}-\bar{\phi}_{N W}+\bar{\phi}_{S W}\right)}{\zeta_{N}-\zeta_{S}}
$$

The flux values and gradients through other faces can be expressed in the same way. It should be also pointed out that the grids in the parametric domain are orthogonal but not necessarily square since they have been arranged based on the knot vectors of the B-spline geometry of the physical space. 


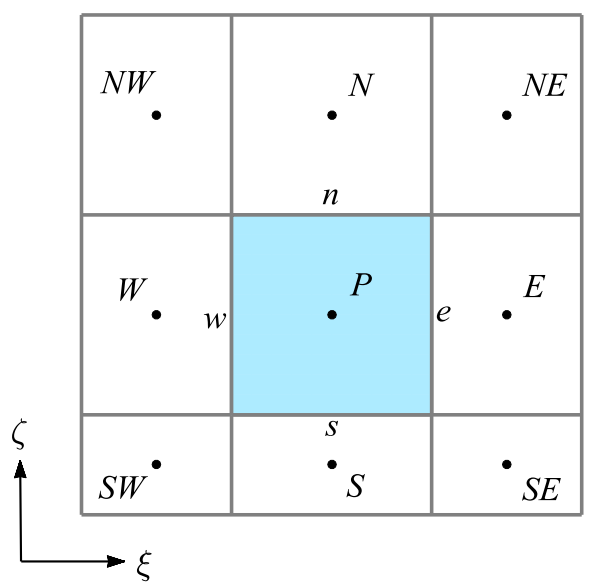

Fig. 2. A control volume centered at $(\mathrm{P})$ and its boundaries and neighbors in the parametric domain

In order to perform the time discretization of the integral formulae of Eq. (19), we employ the standard time marching algorithm. For instance, the continuity equation in the parametric domain can be expressed as:

$$
\int_{\Omega_{0}^{k}} \frac{\bar{\rho}^{(t+1)}-\bar{\rho}^{(t)}}{\Delta t}|J| d \xi d \zeta+\sum_{l=n, e, w, s}\left[\int_{s_{0}^{k, l}}\left(\hat{\rho} \widehat{\boldsymbol{u}}_{l}^{T}\right)^{(t)} J^{-T} \boldsymbol{n}_{0}^{l}|J| d s_{0}\right]=0
$$

where $t$ is the pseudo time step. All other equations follow the same time marching scheme so that by applying the appropriate boundary conditions, the values of field variables in each control volume will be calculated. The choice of $\Delta t$ is, however, of central importance to avoid instability in the analysis. It is also interesting to mention that since the values of $\bar{\phi}$ and $\hat{\phi}_{l}$ are constant in the control volumes, only geometric quantities should be accounted for in the integrals. The integration is then to be performed based on the standard Gauss-Legendre quadrature rule.

Finally, before investigating the two-phase flow in steam turbine cascades, in order to verify the implementation of the isogeometric finite volume method in a well-established wet-steam flow problem, the interested reader is referred to Appendix.

\section{Modeling two-phase flow in steam turbine cascades}

In this section, we present the physical domain of the two-dimensional wet-steam flow in turbine cascades investigating how the respective geometry is constructed and an appropriate grid can be generated. In addition, the numerical results are validated against the experimental data.

\subsection{Physical domain of the problem}

Fig. 3 represents some stationary blades and flow passages in the last stages of an LP steam turbine. This well-known cascade model has been frequently considered for flow simulations in the literature (see, e.g., $[37,41,52,77])$. In order to simulate the wet-steam flow through cascades, one flow passage with periodic 
boundaries, as shown in the figure, is considered. The geometrical specifications of the turbine blades and the boundary conditions at inlet and outlet are listed in Table 2 and Table 3, respectively, taking into account that the outflow is assumed as a supersonic flow. More details regarding the experimental setup and cascade technical data can be found in [77].

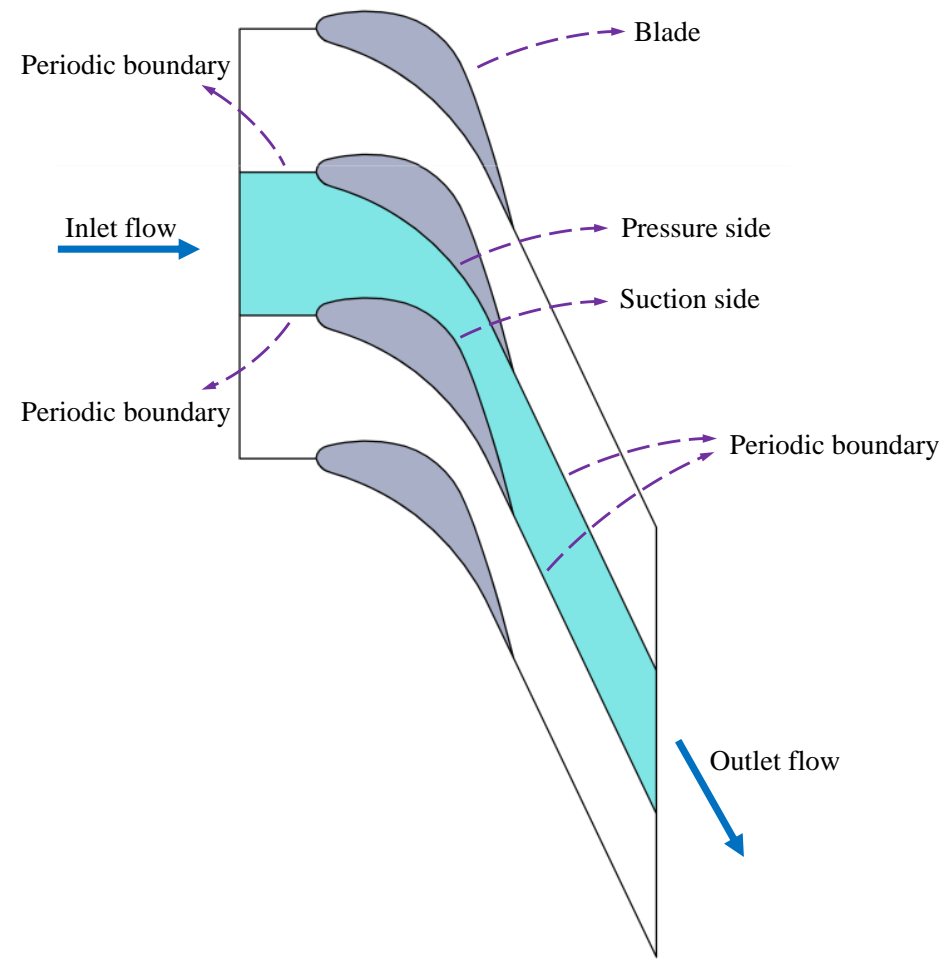

Fig. 3. Physical domain of the flow in cascades (one passage between two blades is highlighted)

Table 2. Geometrical specifications of the blade profile

\begin{tabular}{ccccc}
\hline Length $(\mathrm{mm})$ & Chord $(\mathrm{mm})$ & Pitch $(\mathrm{mm})$ & Axial chord $(\mathrm{mm})$ & Inlet flow angle \\
\hline 76.00 & 35.76 & 18.26 & 25.27 & 0 \\
\hline
\end{tabular}

Table 3. Boundary conditions of the turbine cascade

\begin{tabular}{ccc}
\hline$P_{0, \text { in }}(\mathrm{kPa})$ & $T_{0, \text { in }}=T_{S}\left(P_{0, \text { in }}\right)-8(\mathrm{~K})$ & $P_{\text {out }}=0.48 P_{0, \text { in }}(\mathrm{kPa})$ \\
\hline 172 & 380.66 & 82.56 \\
\hline
\end{tabular}

\subsection{Geometry construction and grid generation}

As stated earlier, the grid generation in the isogeometric finite volume analysis is inherently performed by B-spline representation of the geometric space of the physical domain (see §3.1). For this purpose, the 
position of control points, degrees and knot vectors of the physical domain needs to be extracted from the CAD model of the cascade. It is also important to consider that a basic mesh generation technique is not suitable for flow simulation through cascades since accounting for condensation shock zone and boundary layers in near-the-wall zones needs finer grids in those regions. As a result, a block-structured mesh generation technique (described in, e.g., [78]) integrated with an adaptive mesh refinement based on the standard $h$-refinement technique of the IGA approach (see, e.g., [79]) should be employed. Fig. 4a illustrates three different sections for mesh generation, namely inlet, interior, and outlet sections, as well as zones that need finer grids. The original control points of the respective sections are also depicted in Fig. 4b. Considering a bicubic B-spline surface for each section and based on the B-spline properties described in $\$ 3.1$, the original arrangement of control points will generate a (coarse) grid of size $4 \times 69$ for the entire cascade that should be refined later for achieving reliable results.

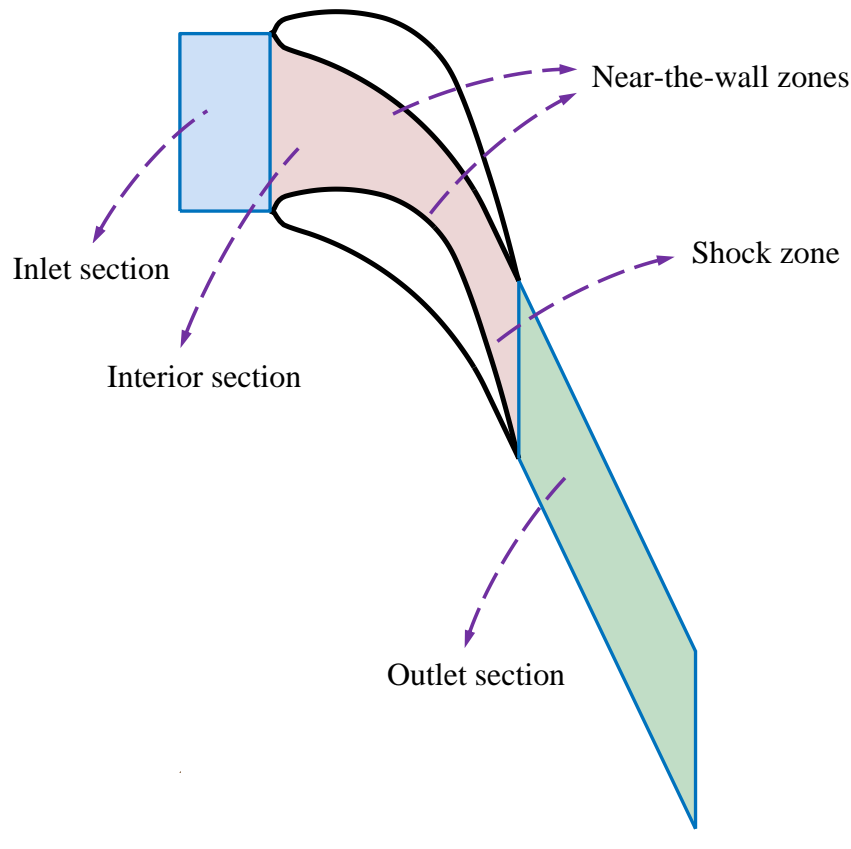

(a)

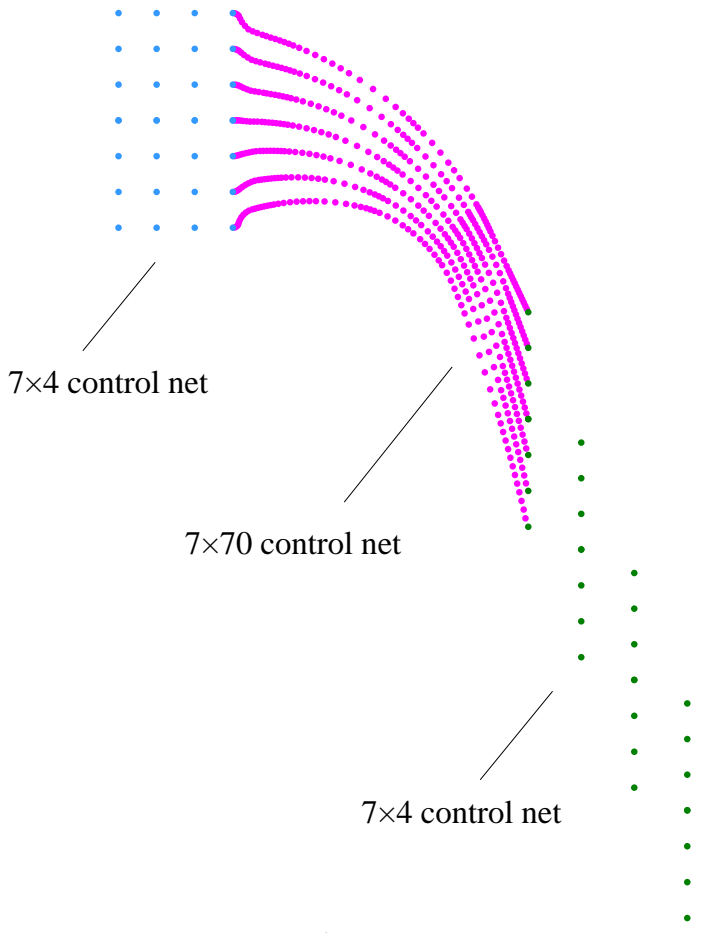

(b)

Fig. 4. (a) Different sections and important zones for grid generation, (b) original control points of the physical domain

\subsection{Validation}

In order to find the suitable grid for flow simulation and validate the accuracy of the numerical modeling, a mesh study with different grid sizes is conducted and the simulated pressure ratios $\left(P / P_{0}\right)$ are compared to experimental data of Bakhtar et al. [37]. In all meshing scenarios, the grid becomes gradually denser near the walls and close to the shock zone. Fig. 5a depicts an $18 \times 115$ grid as the coarsest mesh of this study, which neglects near-the-wall zones, while the finest grid with $48 \times 306$ cells is shown in Fig. $5 \mathrm{~b}$. The numerical results of all studied grids are compared to experimental data as demonstrated in Fig. 6. The 
results show that the grids with higher densities are well consistent with experimental data and predict the location of shock with a sufficient accuracy. The convergence plots of the average pressure error in entire cascade and shock-capturing error in the shock zone (Fig. 7) also confirms that the $48 \times 306$ grid can be selected as the appropriate mesh for further analysis (see Section 5). It should be noted that meshing the leading and trailing edges of airfoils is a challenging task in CFD problems. There may be some extra mesh refinements in the leading/trailing edge (see, e.g., [80, 81]) or some trim/extension in the geometry of the airfoil (see, e.g., $[40,82]$ ) in order to deal with the issue of meshing those regions. In this paper, we used a practical way of geometry extension (see Fig. 5) to reduce the computational cost of unnecessary mesh refinement at the leading edge. This geometry extension, with a shape that is mainly related to our meshing scheme, may add a very little local effect on results; however, it has a negligible effect on the overall outputs of the wet-steam flow simulation through the cascade as shown by our validation against experimental data.

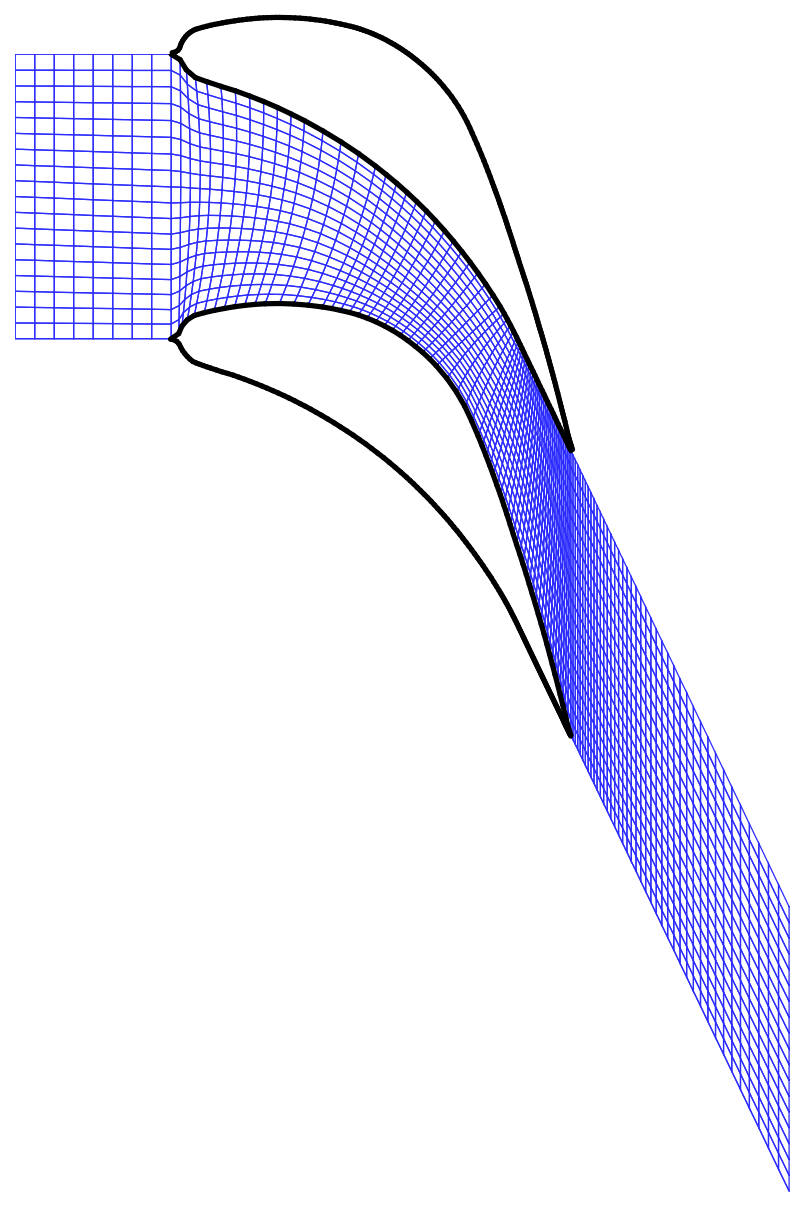

(a)

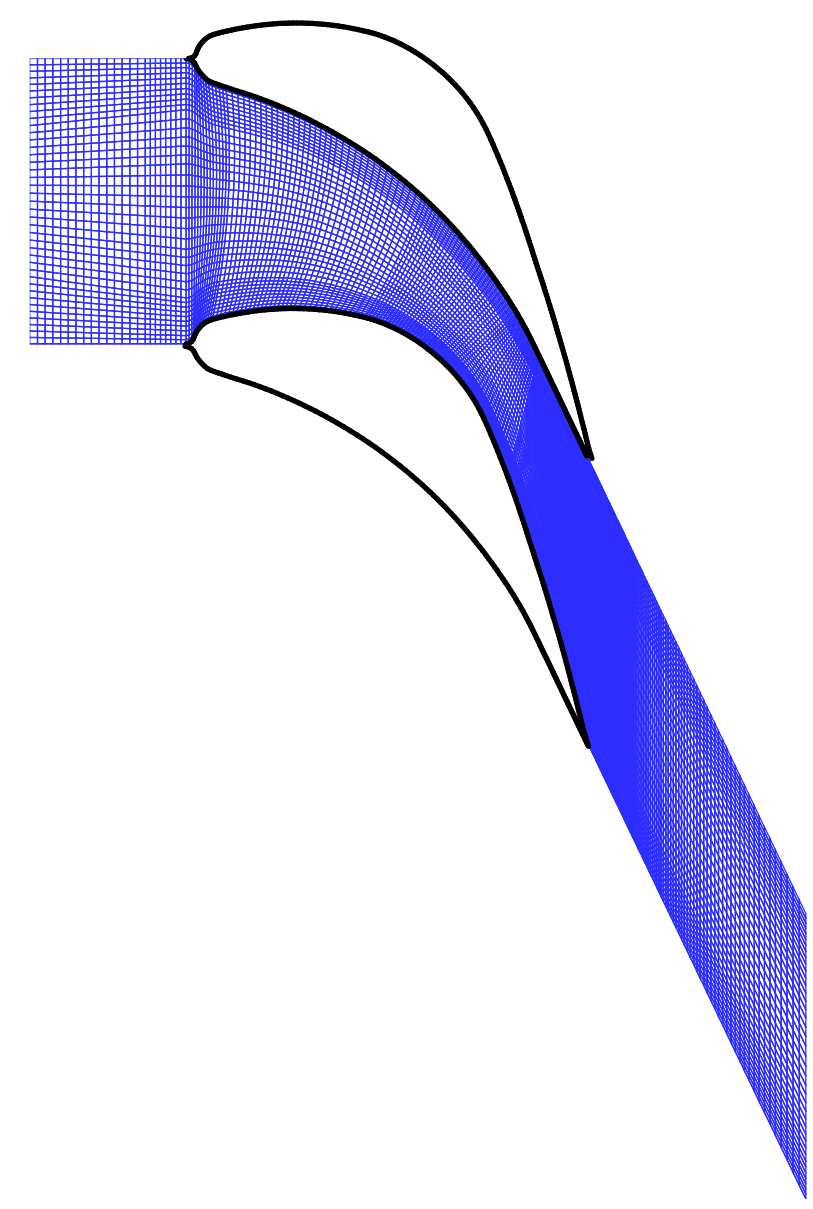

(b)

Fig. 5. (a) Coarsest mesh of this study with $18 \times 115$ grid neglecting near-the-wall zones, (b) finest mesh with $48 \times 306$ grid 


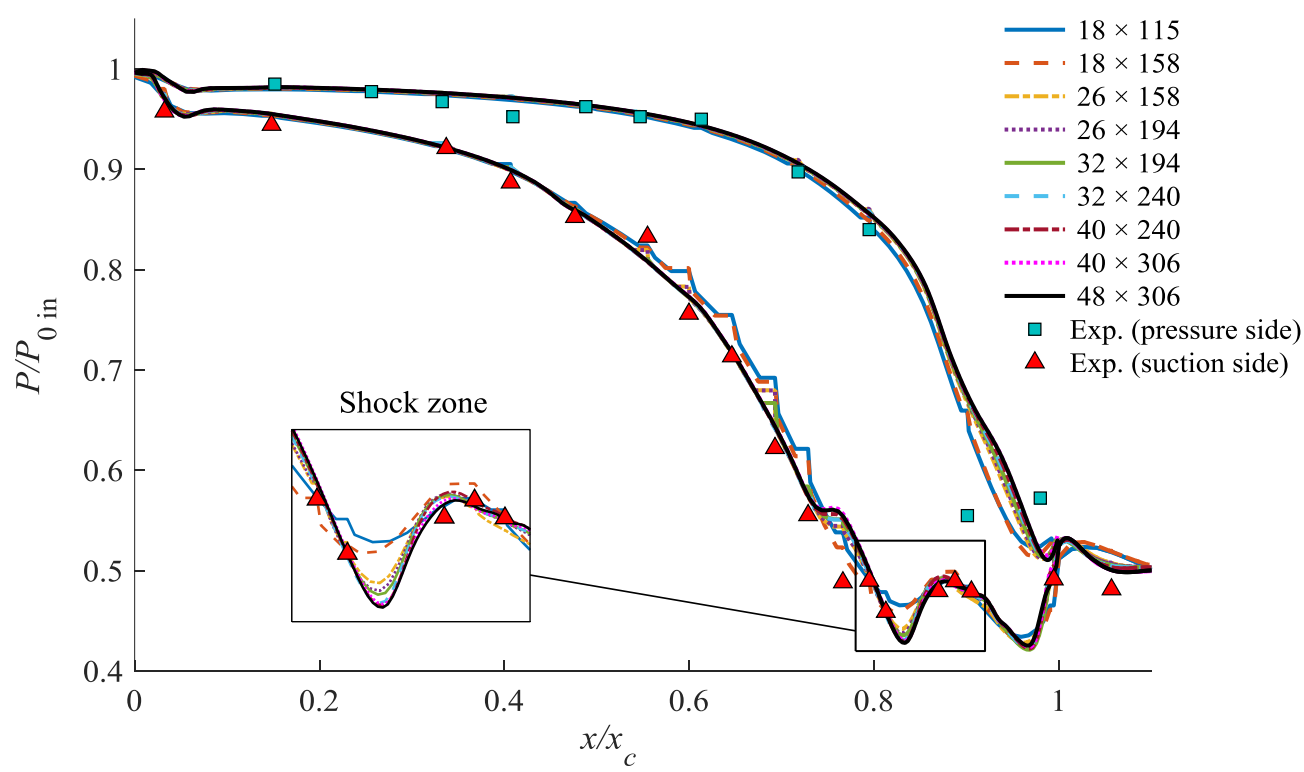

Fig. 6. Pressure ratios in suction and pressure sides of all studied grids compared with experimental data

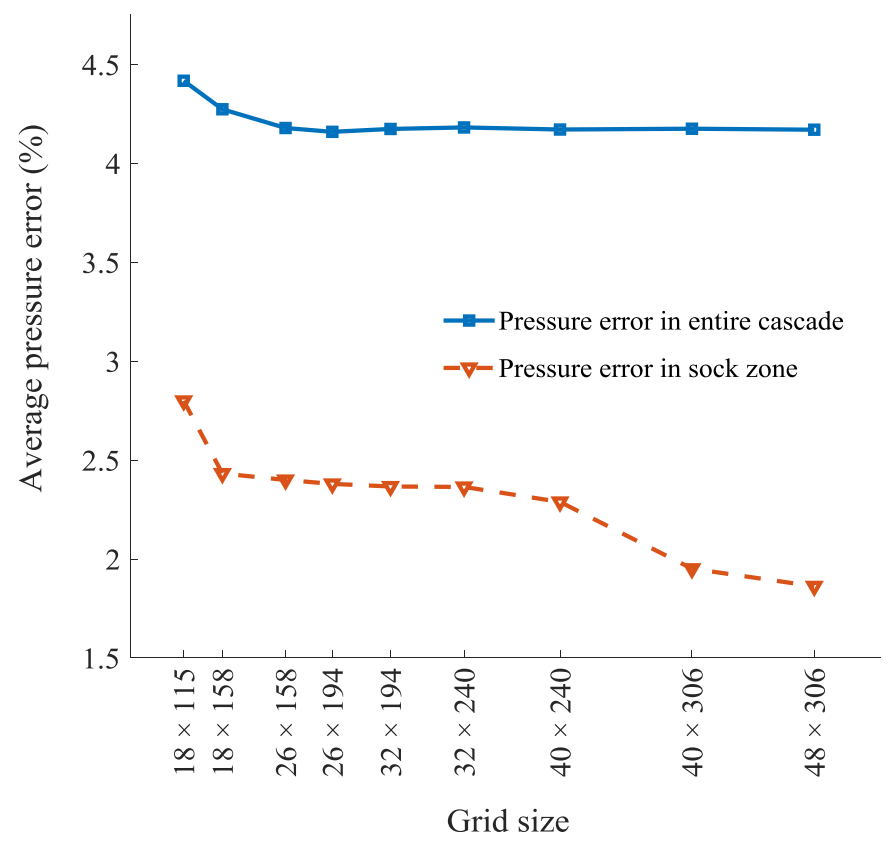

Fig. 7. Convergence graphs of the average pressure error in entire cascade and shock-capturing error in the shock zone

Additionally, in order to compare the accuracy of the employed IGA framework with other approaches that utilize a conventional mesh generation technique, the FVM results of this paper are compared with those obtained by an H-type grid of the same size as reported in Table 4. The comparison reveals that incorporating IGA with FVM has significantly improve the numerical results. 
Table 4. Comparison of numerical errors of the FVM approach: IGA vs. H-type grid

\begin{tabular}{ccc}
\hline & IGA error $(\%)$ & H-grid error $(\%)$ \\
\hline Pressure side & 4.45 & 6.21 \\
Suction side & 3.88 & 5.74 \\
Average error & 4.17 & 5.97 \\
Shock zone & 1.86 & 3.28 \\
Periodical boundary at outlet & 0.55 & 7.34 \\
\hline
\end{tabular}

\section{$5 \quad$ Numerical simulation results}

The numerical simulation results of the wet-stream flow in turbine cascades obtained by the isogeometric finite volume method is presented in this section. In the following graphs, the ratio $x / x_{c}$ is starting from the leading edge $(x=0)$ of the blade and normalized with respect to the $x$-position of the trailing edge $\left(x_{c}\right)$. As seen in Fig. 8a, the density of vapor decreases because of the blade geometry and also due to the condensation shock at $x / x_{c} \approx 0.73$. However, the density of liquid phase rapidly increases up to the maximum value where nucleation starts. Afterwards, the droplets' density reduces slowly and becomes constant by passing the trailing edge. It is worth mentioning that inasmuch as the mass fraction of the liquid phase is far smaller than the vapor phase, no significant variation could be seen in the density graph of the mixture after nucleation (Fig. 8b). The contour of the mixture density is also shown by Fig. 8c.

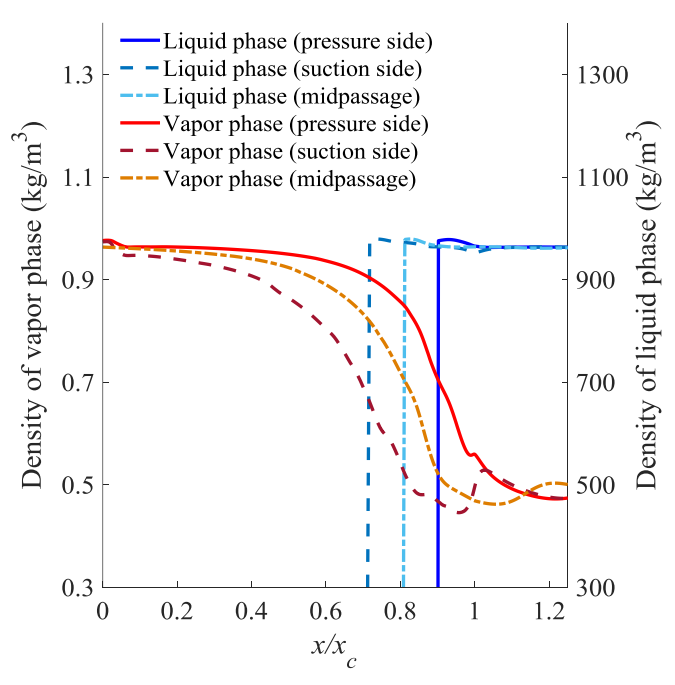

(a)

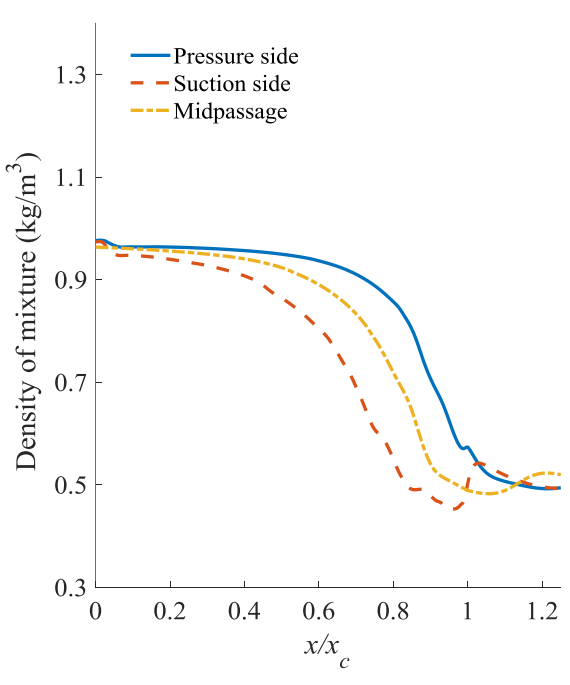

(b)

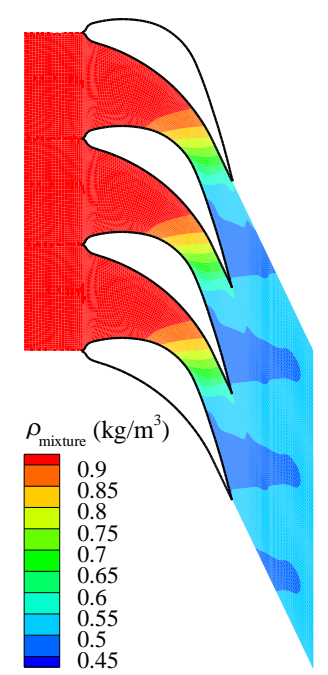

(c)

Fig. 8. Density distribution in cascades: (a) density of liquid and vapor phases, (b) and (c) density of mixture

Fig. 9 illustrates the absolute velocity of the wet-steam flow inside the passage between stationary cascades of steam turbine blades. Due to the blade geometry, the pressure reduces and therefore, the velocity has an increasing trend until the condensation shock occurred (Fig. 9a). At this place, the rate of increase in the mixture velocity is reduced, which is due to the condensation shock and droplets' nucleation. Afterwards, 
the velocity continues increasing up to $x / x_{c}=1$ at trailing edge where it reduces sharply because of the aerodynamic shock and also the inflection of the pressure surface shock on the suction surface. The variation of velocity through the computational domain of cascades is shown in Fig. $9 \mathrm{~b}$.

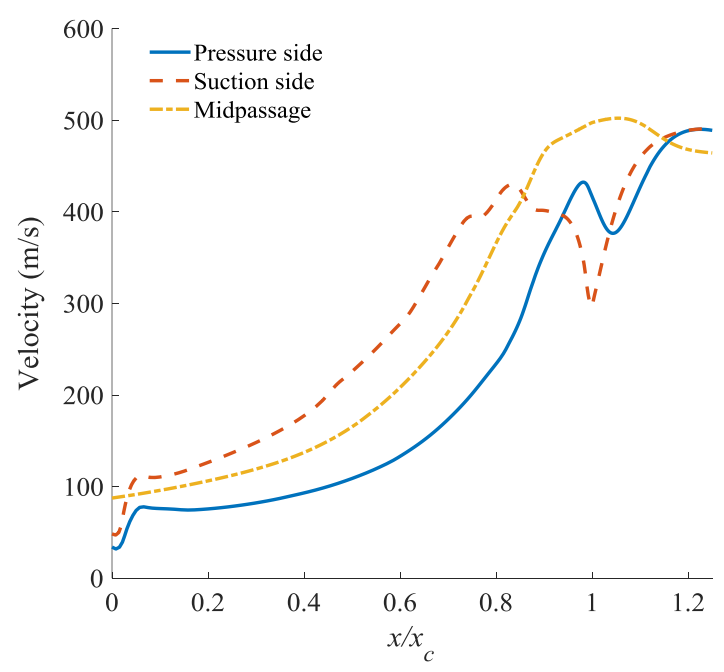

(a)

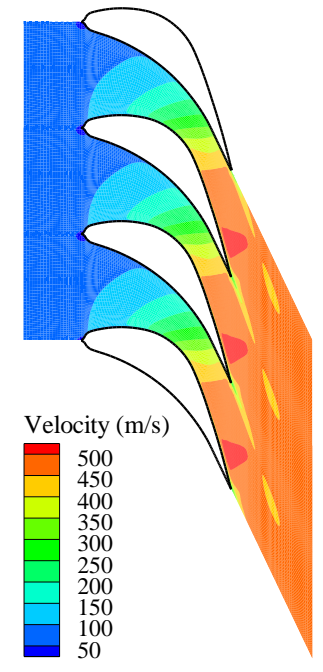

(b)

Fig. 9. Velocity distribution of the wet-steam flow: (a) absolute velocity on the suction side, pressure side and midpassage,

(b) velocity contour in entire cascade

Fig. 10 demonstrates the variations in temperature of liquid and vapor phases. As can be seen in Fig. 10a, the vapor temperature reduces because of the non-equilibrium conditions that governs the wet-steam flow. Then, by nucleating and appearing the first droplets, the temperature of liquid phase increases rapidly. Simultaneously, the vapor temperature has an increasing trend, which is due to the heat transfer of droplets to the continuous phase. Finally, the temperatures of both phases become equal which indicates the establishment of equilibrium conditions. The contours of liquid and vapor temperature are represented in Fig. 10b and Fig. 10c, respectively.

An important property to measure in the wet-steam flow analysis is the degree of supercooling that is defined as the difference of the vapor and the saturation temperatures corresponding to the pressure of flow $T_{S}(P)-T_{v}$. As illustrated in Fig. 11a, the diagram of supercooling degree has a steady trend until the moment in which the condensation shock occurs on the suction surface $\left(x / x_{c} \approx 0.73\right)$ and inflected on the pressure surface at $x / x_{c} \approx 0.92$. At the mentioned places, the supercooling degree reaches the highest values of $31.4 \mathrm{~K}$. However, it is worth noting that the highest value of the supercooling degree occurs inside the cascade at the condensation zone that is equal to the $33.78 \mathrm{~K}$. Afterwards, the supercooling degree sharply drops due to the difference in pressure. Finally, the variations decrease because of the reduction in difference between the vapor and droplets temperatures. As shown in the supercooling contour of Fig. 11b, the fluid is diverted rapidly from the thermodynamic equilibrium at the condensation zone and returns to the equilibrium after nucleating and heat transferring between phases. 


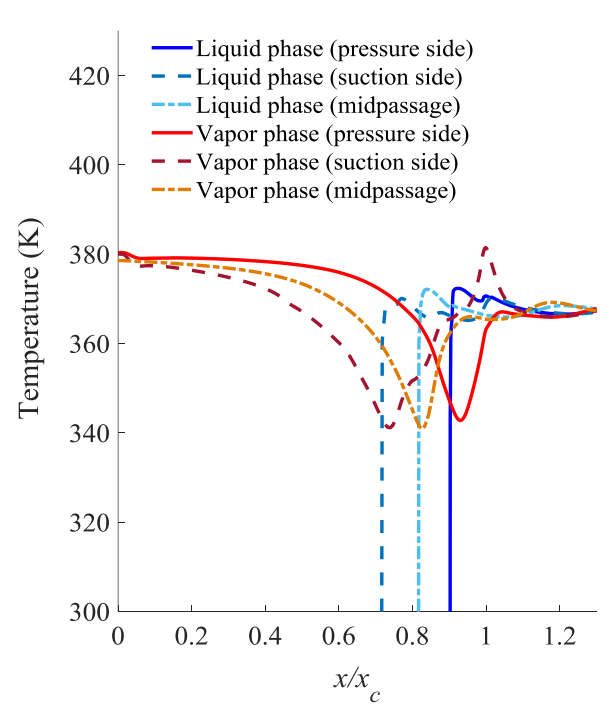

(a)

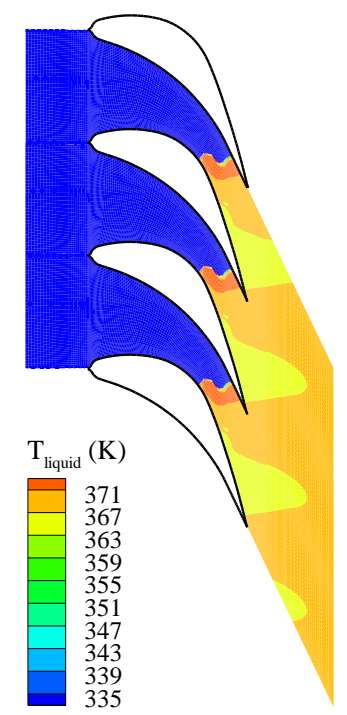

(b)

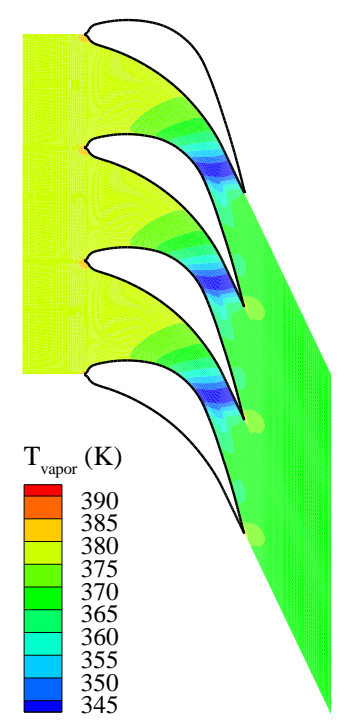

(c)

Fig. 10. (a) Temperature graphs of the liquid and vapor phases along the streamlines, (b) and (c) temperature contours of liquid and vapor, respectively

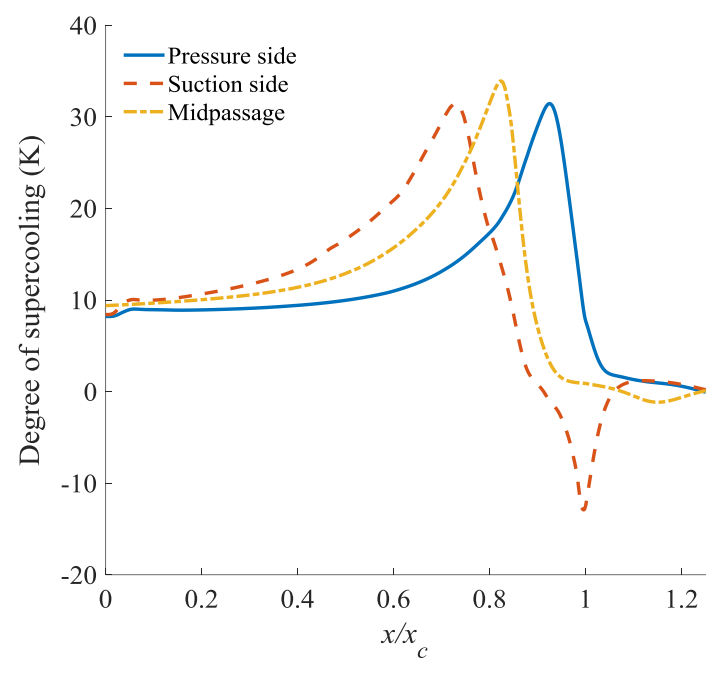

(a)

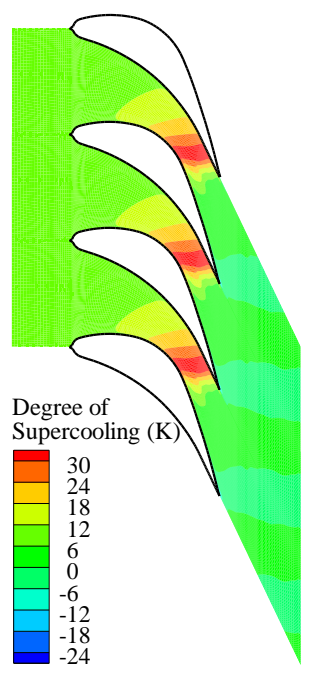

(b)

Fig. 11. Degree of supercooling: (a) along the streamlines, (b) around the blade cascades

The mass fraction of the liquid phase $\beta$ is another important feature to investigate in the wet-steam flow analysis. The mass fraction of the nucleated droplets increases by beginning the nucleation process and become almost constant after leaving the condensation zone. As shown in Fig. 12a, the average droplet radius $\bar{r}_{d}$ also starts increasing at $x / x_{c} \approx 0.84$ on the midpassage streamline and reaches a maximum near the trailing edge. It is important to mention that a reduction in wetness fraction of the wet-steam flow can be observed by passing the trailing edge where the aerodynamic shock occurs. The reason is that the evaporation temperature increases in that region and causes a decrease in the value of $\beta$. Fig. 12b also represents the wetness fraction contour. 


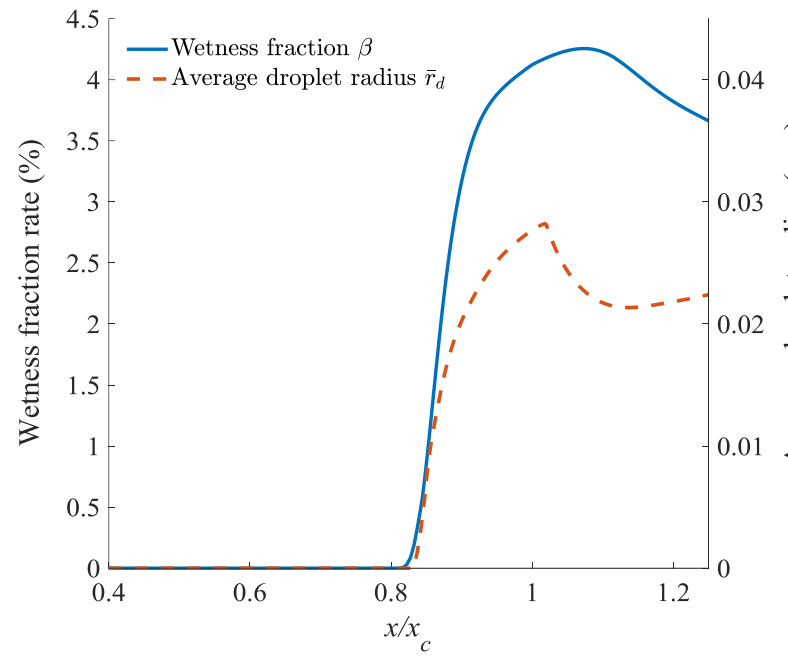

(a)

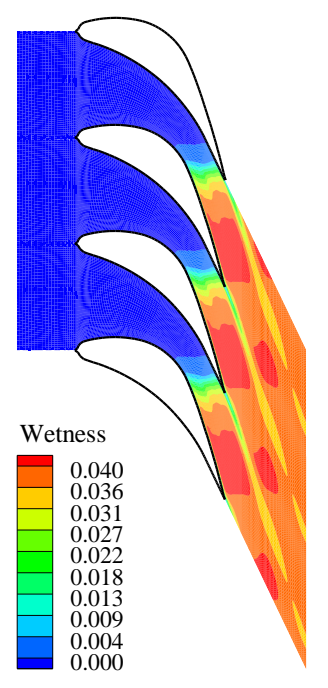

(b)

Fig. 12. (a) Distribution of wetness fraction and average droplet radius along the midpassage, (b) wetness fraction contour around the blade cascades

Finally, the turbulence parameters (i.e., the kinetic energy $k$ and the rate of dissipation $\omega$ ) are depicted in Fig. 13. As shown in the figure, the kinetic energy increases through the passage since the velocity of the wet-steam increases along the blade and therefore, the flow gradually becomes turbulent. The dissipation rate also clearly changes along the blade that is because of the turbulent condition of flow. In addition, the dissipation rate decreases near the trailing edge under the influence of velocity drop due to the aerodynamic shock.

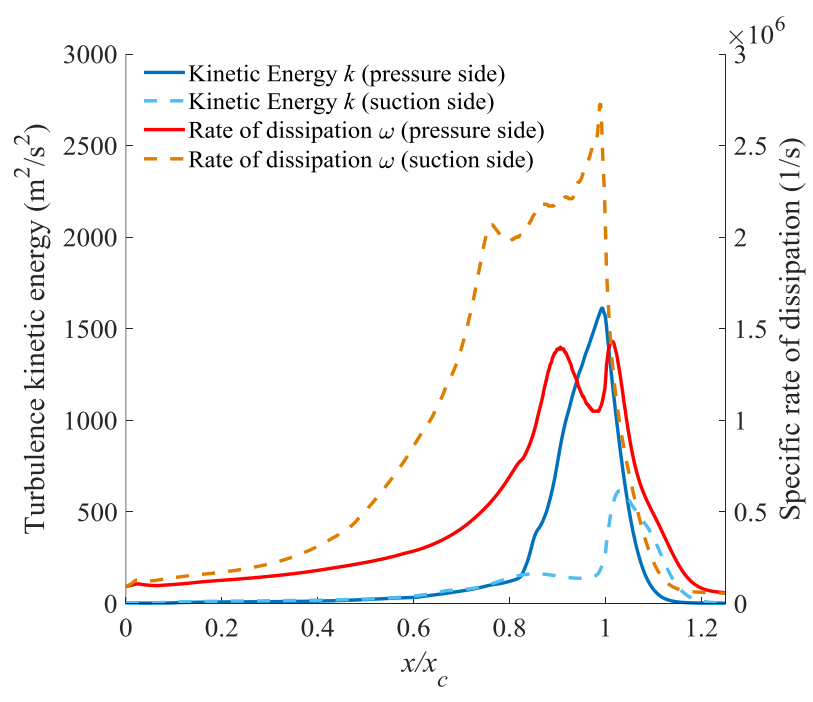

(a)

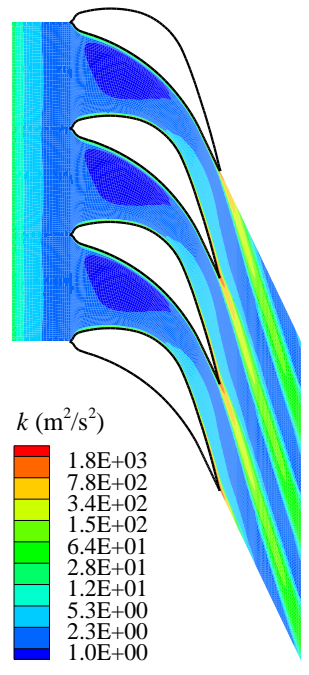

(b)

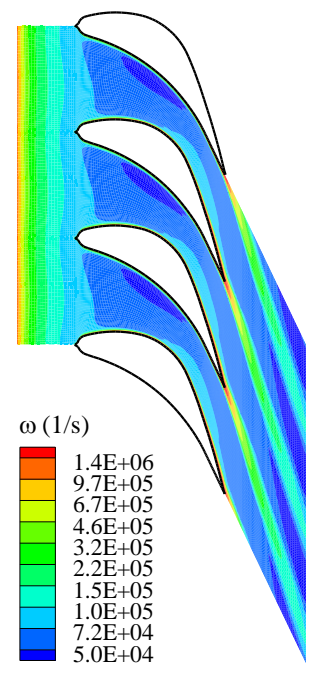

(c)

Fig. 13. Kinetic energy and dissipation rate on the pressure and suction surfaces (a) and through the blade cascades (b) 


\section{Conclusions}

This paper presents an isogeometric finite volume analysis with the aim of having a reliable solution for the two-phase flow simulation through the stationary cascades of low-pressure steam turbines. Based on the presented formulation and results of this paper, the following conclusions can be drawn:

- Utilizing the isogeometric framework, a more compatible grid with the geometry of the steam turbine cascades is generated.

- Computed results are validated against experimental measurements based on static pressure distribution.

- The numerical results show a significant improvement in comparison with the H-type grid of the same size as a conventional grid generation technique.

- The condensation shock locations are accurately predicted and tracked.

- Main specifications of a wet-steam flow like, e.g., wetness, degree of supercooling, temperature and density of the liquid and vapor phases, as well as the flow velocity and turbulence parameters are computed using the employed isogeometric framework.

\section{Acknowledgements}

Ali Hashemian was partially funded by the BCAM "Severo Ochoa" accreditation of excellence (SEV-20170718), and the Basque Government through the BERC 2018-2021 program. The authors would also like to thank the anonymous reviewers for their constructive suggestions that helped to improve the paper.

\section{Appendix. Verifying isogeometric FVM by a well-established example}

In order to verify the implementation of the isogeometric finite volume method in wet-steam flow problems, a well-established example, presented by Moore et al. [83], is herein considered. In this regard, three convergent-divergent nozzles (A, B, and C) with geometrical specifications reported in Fig. A.1 and Table A.1 are studied. The inlet conditions of the wet-steam flow for different nozzles are also given in Table A.2.

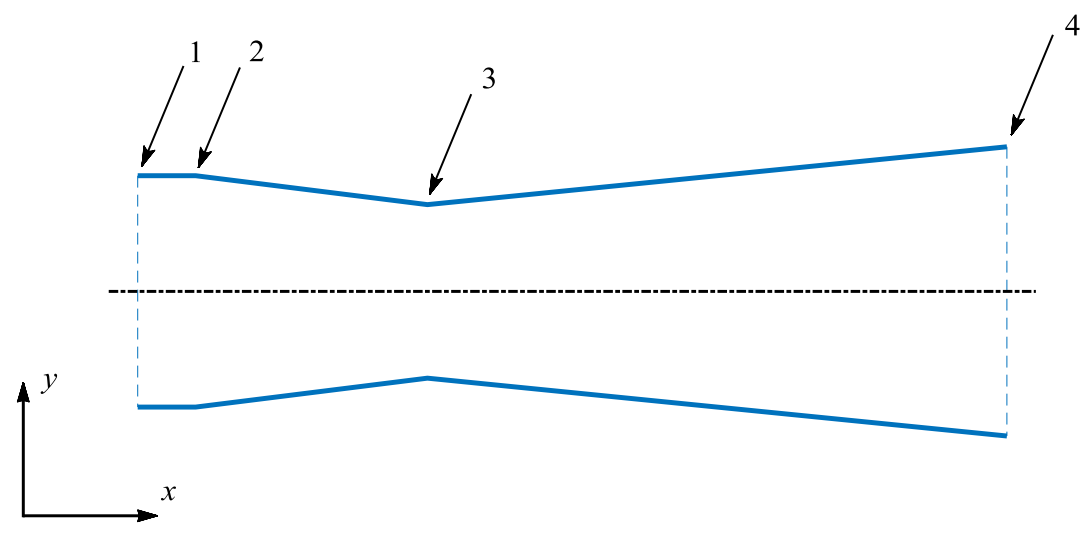

Fig. A.1. Geometry of studied convergent-divergent nozzles 
Table A.1. Coordinates of different sections shown in Fig. A.1 for nozzles A to C [83]

\begin{tabular}{ccccc}
\hline \multirow{2}{*}{ Position } & \multirow{2}{*}{$x$-coordinate $(\mathrm{m})$} & Nozzle A & Nozzle B & Nozzle C \\
\hline 1 & -0.25 & 0.03785 & 0.05635 & 0.06985 \\
2 & -0.2 & 0.03785 & 0.05635 & 0.06985 \\
3 & 0 & 0.0315 & 0.05 & 0.0635 \\
4 & 0.5 & 0.057 & 0.072 & 0.074 \\
\hline
\end{tabular}

Table A.2. Inlet conditions of the wet-steam flow for different nozzles

\begin{tabular}{lccc}
\hline & Nozzle A & Nozzle B & Nozzle C \\
\hline$P_{0, \text { in }}(\mathrm{kPa})$ & 25 & 25 & 25 \\
$T_{0, \text { in }}=T_{S}\left(P_{0, \text { in }}\right)-8(\mathrm{~K})$ & 354.6 & 357.6 & 358.6 \\
\hline
\end{tabular}

The nozzle geometry in this example is symmetric about the centerline and, therefore, only one half the model is studied. Furthermore, it should be noted that the nozzle geometry is not curved and all control volumes are straight. Fig. A.2 depicts the meshed geometry of the nozzles A to C, noting that the mesh is finer near the walls and also in the diverging region after the throat (shock zone). The refinement level for each nozzle is obtained by the convergence study shown in

Fig. A.3. Finally, the static pressure distributions computed by isogeometric FVM along the centerline in the diverging zone of nozzles are verified by experimental data reported in [83] as shown in Fig. A.4.

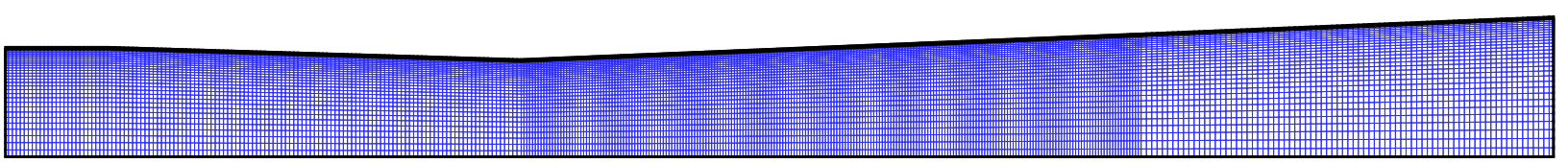

Nozzle A

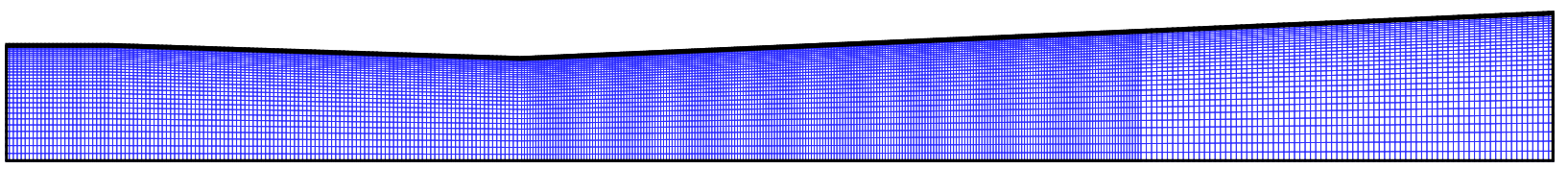

Nozzle B

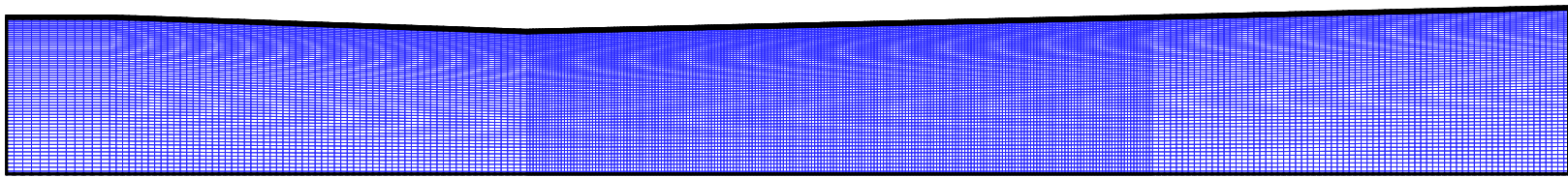

Nozzle C

Fig. A.2. Generated grids for different studied nozzles 


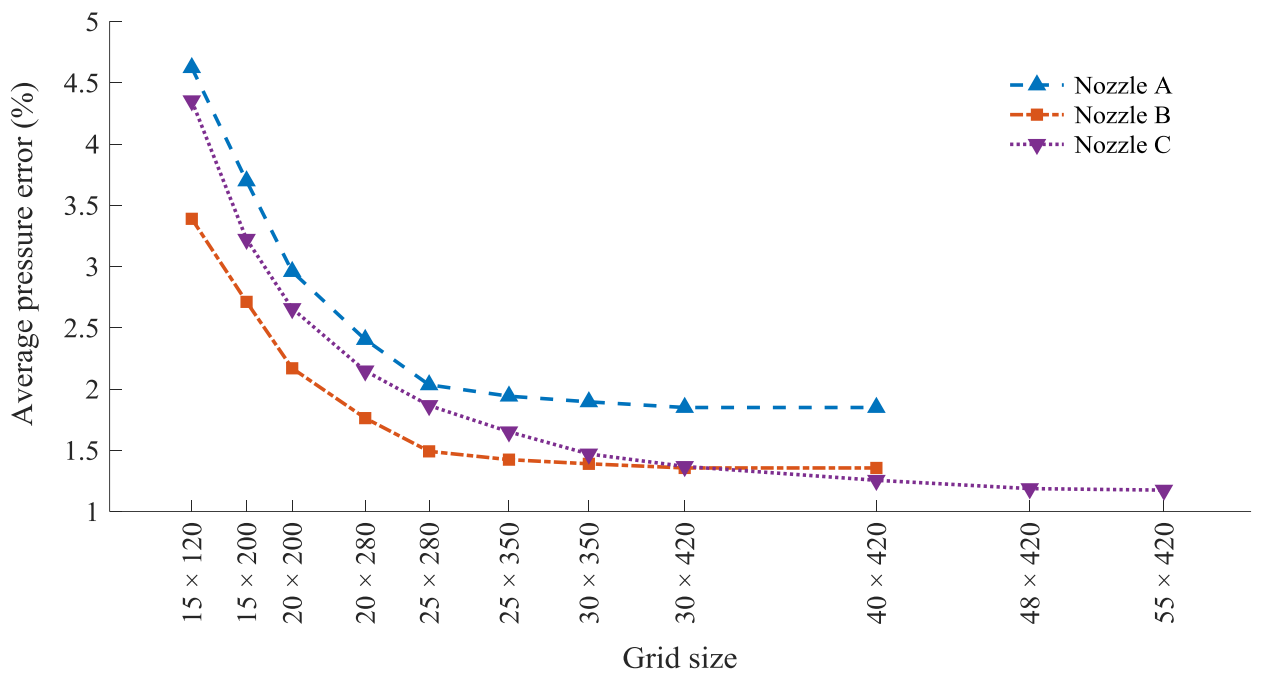

Fig. A.3. Convergence study of the average pressure error in nozzles A to $\mathrm{C}$ (the relative errors are obtained by comparing the computed pressures with reference values reported in Moore et al. [83])

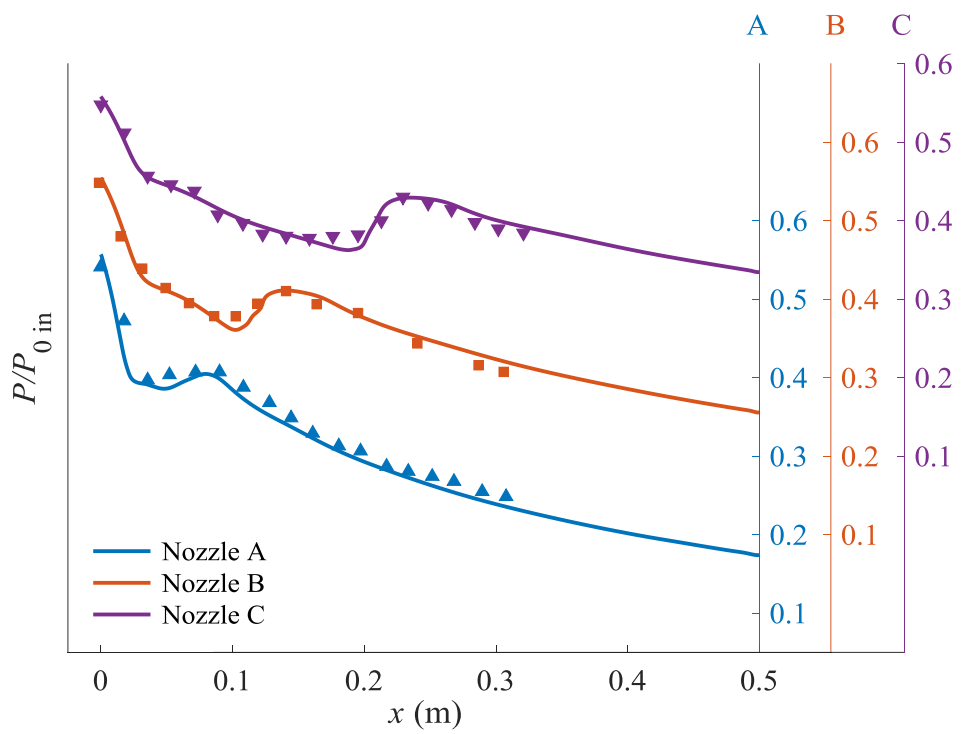

Fig. A.4. Comparison of the static pressure along the centerline in the diverging zone of nozzles A to C: distributions computed by isogeometric FVM (solid lines) vs. experimental data (markers) reported by Moore et al. [83] (for interpretation of the references to color in this figure legend, the reader is referred to the web version of this article)

\section{References}

[1] T.J.R. Hughes, J.A. Cottrell, Y. Bazilevs, Isogeometric analysis: CAD, finite elements, NURBS, exact geometry and mesh refinement, Computer Methods in Applied Mechanics and Engineering, 194 (2005) 4135-4195.

[2] J. Sogn, S. Takacs, Robust multigrid solvers for the biharmonic problem in isogeometric analysis, Computers \& Mathematics with Applications, 77 (2019) 105-124.

[3] S.E. Moore, Discontinuous Galerkin Isogeometric Analysis for the biharmonic equation, Computers \& Mathematics with Applications, 76 (2018) 673-685. 
[4] D. Garcia, D. Pardo, L. Dalcin, V.M. Calo, Refined Isogeometric Analysis for a preconditioned conjugate gradient solver, Computer Methods in Applied Mechanics and Engineering, 335 (2018) 490-509.

[5] D. Garcia, D. Pardo, L. Dalcin, M. Paszyński, N. Collier, V.M. Calo, The value of continuity: Refined isogeometric analysis and fast direct solvers, Computer Methods in Applied Mechanics and Engineering, 316 (2017) 586-605.

[6] R.N. Simpson, Z. Liu, R. Vázquez, J.A. Evans, An isogeometric boundary element method for electromagnetic scattering with compatible B-spline discretizations, Journal of Computational Physics, 362 (2018) 264-289.

[7] A. Buffa, G. Sangalli, R. Vázquez, Isogeometric methods for computational electromagnetics: B-spline and T-spline discretizations, Journal of Computational Physics, 257 (2014) 1291-1320.

[8] Y. Guo, M. Ruess, Weak Dirichlet boundary conditions for trimmed thin isogeometric shells, Computers \& Mathematics with Applications, 70 (2015) 1425-1440.

[9] G. Balduzzi, S. Morganti, F. Auricchio, A. Reali, Non-prismatic Timoshenko-like beam model: Numerical solution via isogeometric collocation, Computers \& Mathematics with Applications, 74 (2017) 1531-1541.

[10] R. Bouclier, T. Elguedj, A. Combescure, On the development of NURBS-based isogeometric solid shell elements: 2D problems and preliminary extension to 3D, Computational Mechanics, 52 (2013) 10851112.

[11] H. Gomez, T.J.R. Hughes, X. Nogueira, V.M. Calo, Isogeometric analysis of the isothermal NavierStokes-Korteweg equations, Computer Methods in Applied Mechanics and Engineering, 199 (2010) 18281840.

[12] B.S. Hosseini, S. Turek, M. Möller, C. Palmes, Isogeometric Analysis of the Navier-Stokes-CahnHilliard equations with application to incompressible two-phase flows, Journal of Computational Physics, 348 (2017) 171-194.

[13] Y. Bazilevs, V.M. Calo, T.J.R. Hughes, Y. Zhang, Isogeometric fluid-structure interaction: theory, algorithms, and computations, Computational Mechanics, 43 (2008) 3-37.

[14] L. Yang, One-fluid formulation for fluid-structure interaction with free surface, Computer Methods in Applied Mechanics and Engineering, 332 (2018) 102-135.

[15] M. Yoon, S.-H. Ha, S. Cho, Isogeometric shape design optimization of heat conduction problems, International Journal of Heat and Mass Transfer, 62 (2013) 272-285.

[16] Z.-P. Wang, S. Turteltaub, M. Abdalla, Shape optimization and optimal control for transient heat conduction problems using an isogeometric approach, Computers \& Structures, 185 (2017) 59-74.

[17] T.J.R. Hughes, J.A. Evans, A. Reali, Finite element and NURBS approximations of eigenvalue, boundary-value, and initial-value problems, Computer Methods in Applied Mechanics and Engineering, 272 (2014) 290-320.

[18] D. Wang, Q. Liang, H. Zhang, A superconvergent isogeometric formulation for eigenvalue computation of three dimensional wave equation, Computational Mechanics, 57 (2016) 1037-1060.

[19] S. Dykas, W. Wróblewski, H. Łukowicz, Prediction of losses in the flow through the last stage of lowpressure steam turbine, International Journal for Numerical Methods in Fluids, 53 (2007) 933-945.

[20] M. Mahpeykar, E. Lakzian, E. Amirirad, Reduction of thermodynamic losses in a supersonic nucleating steam nozzle by spraying water droplets, Scientia Iranica, 16 (2009) 253-262. 
[21] M.R. Mahpeykar, A.R. Teymourtash, E. Amiri Rad, Theoretical investigation of effects of local cooling of a nozzle divergent section for controlling condensation shock in a supersonic two-phase flow of steam, Meccanica, 48 (2013) 815-827.

[22] M. Häfele, J. Starzmann, M. Grübel, M. Schatz, D. Vogt, R. Drozdowski, L. Völker, Numerical investigation of the impact of part-span connectors on aero-thermodynamics in a low pressure industrial steam turbine, in: ASME Turbo Expo 2014: Turbine Technical Conference and Exposition, American Society of Mechanical Engineers, (2014) V01BT27A004-014.

[23] M. Grübel, J. Starzmann, M. Schatz, T. Eberle, D. Vogt, F. Sieverding, Two-Phase Flow Modeling and Measurements in Low-Pressure Turbines-Part I: Numerical Validation of Wet Steam Models and Turbine Modeling, Journal of Engineering for Gas Turbines and Power, 137 (2015) 042602-042611.

[24] A. White, J. Young, P. Walters, Experimental validation of condensing flow theory for a stationary cascade of steam turbine blades, Philosophical Transactions of the Royal Society of London. Series A: Mathematical, Physical and Engineering Sciences, 354 (1996) 59-88.

[25] H. Mashmoushy, M.R. Mahpeykar, F. Bakhtar, Studies of nucleating and wet steam flows in twodimensional cascades, Proceedings of the Institution of Mechanical Engineers, Part C: Journal of Mechanical Engineering Science, 218 (2004) 843-858.

[26] W. Wróblewski, S. Dykas, A. Gardzilewicz, M. Kolovratnik, Numerical and experimental investigations of steam condensation in LP part of a large power turbine, Journal of Fluids Engineering, 131 (2009) 041301-041311.

[27] A.H. Yousif, A.M. Al-Dabagh, R.C. Al-Zuhairy, Non-equilibrium spontaneous condensation in transonic steam flow, International Journal of Thermal Sciences, 68 (2013) 32-41.

[28] M. Schatz, T. Eberle, M. Grübel, J. Starzmann, D. Vogt, N. Sürken, Two-Phase Flow Modeling and Measurements in Low-Pressure Turbines-Part II: Turbine Wetness Measurement and Comparison to Computational Fluid Dynamics-Predictions, Journal of Engineering for Gas Turbines and Power, 137 (2015) 042603-042609.

[29] S. Dykas, M. Majkut, M. Strozik, K. Smołka, Experimental study of condensing steam flow in nozzles and linear blade cascade, International Journal of Heat and Mass Transfer, 80 (2015) 50-57.

[30] F. Bakhtar, K. Zidi, On the self diffusion of water vapour, Proceedings of the Institution of Mechanical Engineers, Part C: Journal of Mechanical Engineering Science, 199 (1985) 159-164.

[31] S. Sinha, B.E. Wyslouzil, G. Wilemski, Modeling of H2O/D2O condensation in supersonic nozzles, Aerosol Science and Technology, 43 (2009) 9-24.

[32] E. Lakzian, A. Masjedi, Slip effects on the exergy loss due to irreversible heat transfer in a condensing flow, International Journal of Exergy, 14 (2014) 22-37.

[33] Y. Liao, D. Lucas, A literature review on mechanisms and models for the coalescence process of fluid particles, Chemical Engineering Science, 65 (2010) 2851-2864.

[34] E. Lakzian, S. Shaabani, Analytical investigation of coalescence effects on the exergy loss in a spontaneously condensing wet-steam flow, International Journal of Exergy, 16 (2015) 383-403.

[35] N. Sakai, T. Harada, Y. Imai, Numerical study of partial admission stages in steam turbine, JSME International Journal Series B Fluids and Thermal Engineering, 49 (2006) 212-217.

[36] J. Joseph, S. Subramanian, K. Vigney, B. Prasad, D. Biswas, Thermodynamic wetness loss calculation in nozzle and turbine cascade: nucleating steam flow, Heat and Mass Transfer, 54 (2018) 2521-2531. 
[37] F. Bakhtar, M.R. Mahpeykar, K. Abbas, An investigation of nucleating flows of steam in a cascade of turbine blading-theoretical treatment, Journal of Fluids Engineering, 117 (1995) 138-144.

[38] F. Bakhtar, M. Zamri, J. Rodriguez-Lelis, A comparative study of treatment of two-dimensional twophase flows of steam by a Runge-Kutta and by Denton's methods, Proceedings of the Institution of Mechanical Engineers, Part C: Journal of Mechanical Engineering Science, 221 (2007) 689-706.

[39] M.S. Mirhoseini, M. Boroomand, Multi-objective optimization of hot steam injection variables to control wetness parameters of steam flow within nozzles, Energy, 141 (2017) 1027-1037.

[40] F. Giacomelli, F. Mazzelli, A. Milazzo, CFD modelling of the condensation inside a cascade of steam turbine blades: comparison with an experimental test case, Energy Procedia, 126 (2017) 730-737.

[41] M. Vatanmakan, E. Lakzian, M.R. Mahpeykar, Investigating the entropy generation in condensing steam flow in turbine blades with volumetric heating, Energy, 147 (2018) 701-714.

[42] C. Heinrich, B. Simeon, S. Boschert, A finite volume method on NURBS geometries and its application in isogeometric fluid-structure interaction, Mathematics and Computers in Simulation, 82 (2012) 16451666.

[43] W.G. Courtney, Remarks on homogeneous nucleation, The Journal of Chemical Physics, 35 (1961) 2249-2250.

[44] A. Kantrowitz, Nucleation in very rapid vapor expansions, The Journal of chemical physics, 19 (1951) 1097-1100.

[45] J. Young, Two-dimensional, nonequilibrium, wet-steam calculations for nozzles and turbine cascades, Journal of Turbomachinery, 114 (1992) 569-579.

[46] R. Peyret, T.D. Taylor, Computational Methods for Fluid Flow, Springer-Verlag, New York, NY, 1983.

[47] A. Novotny, H. Petzeltová, Weak Solutions for the Compressible Navier-Stokes Equations: Existence, Stability, and Longtime Behavior, in: Y. Giga, A. Novotny (Eds.) Handbook of Mathematical Analysis in Mechanics of Viscous Fluids, Springer International Publishing, New York, NY, (2017).

[48] Y. Patel, G. Patel, T. Turunen-Saaresti, Influence of turbulence modelling on non-equilibrium condensing flows in nozzle and turbine cascade, International Journal of Heat and Mass Transfer, 88 (2015) 165-180.

[49] K. Ishizaka, A high-resolution numerical method for transonic non-equilibrium condensation flow through a steam turbine cascade, in: 6th International Symposium on Computational Fluid Dynamics, Lake Tahoe, USA, (1995) 479-484.

[50] X.-D. Wang, J.-L. Dong, T. Wang, J.-Y. Tu, Numerical analysis of spontaneously condensing phenomena in nozzle of steam-jet vacuum pump, Vacuum, 86 (2012) 861-866.

[51] F. Bakhtar, M.R. Mahpeykar, On the performance of a cascade of turbine rotor tip section blading in nucleating steam Part 3: theoretical treatment, Proceedings of the Institution of Mechanical Engineers, Part C: Journal of Mechanical Engineering Science, 211 (1997) 195-210.

[52] F. Bakhtar, J. Young, A. White, D. Simpson, Classical nucleation theory and its application to condensing steam flow calculations, Proceedings of the Institution of Mechanical Engineers, Part C: Journal of Mechanical Engineering Science, 219 (2005) 1315-1333.

[53] M. Kermani, A. Gerber, A general formula for the evaluation of thermodynamic and aerodynamic losses in nucleating steam flow, International journal of heat and mass transfer, 46 (2003) 3265-3278. 
[54] J. Young, The spontaneous condensation of steam in supersonic nozzle, Physico Chemical Hydrodynamics, 3 (1982) 57-82.

[55] B. Nikkhahi, M. Shams, M. Ziabasharhagh, A numerical investigation of two-phase steam flow around a 2-D turbine's rotor tip, International Communications in Heat and Mass Transfer, 36 (2009) 632-639.

[56] J. Young, An equation of state for steam for turbomachinery and other flow calculations, Journal of Engineering for Gas Turbines and Power, 110 (1988) 1-7.

[57] D.C. Wilcox, Reassessment of the scale-determining equation for advanced turbulence models, AIAA Journal, 26 (1988) 1299-1310.

[58] D.C. Wilcox, Formulation of the k- $\omega$ Turbulence Model Revisited, AIAA Journal, 46 (2008) 28232838.

[59] A. Hashemian, S.F. Hosseini, S.N. Nabavi, Kinematically Smoothing Trajectories by NURBS Reparameterization - An Innovative Approach, Advanced Robotics, 31 (2017) 1296-1312.

[60] S.F. Hosseini, A. Hashemian, B. Moetakef-Imani, S. Hadidimoud, Isogeometric Analysis of Free-form Timoshenko Curved Beams Including the Nonlinear Effects of Large Deformations, Acta Mechanica Sinica, 34 (2018) 728-743.

[61] S.F. Hosseini, A. Hashemian, A. Reali, On the application of curve reparameterization in isogeometric vibration analysis of free-from curved beams, Computers \& Structures, 209 (2018) 117-129.

[62] O. Weeger, B. Narayanan, M.L. Dunn, Isogeometric shape optimization of nonlinear, curved 3D beams and beam structures, Computer Methods in Applied Mechanics and Engineering, 345 (2019) 26-51.

[63] A. Hashemian, S.F. Hosseini, Nonlinear bifurcation analysis of statically loaded free-form curved beams using isogeometric framework and pseudo-arclength continuation, International Journal of NonLinear Mechanics, 113 (2019) 1-16.

[64] H. Saghi, A. Hashemian, Multi-dimensional NURBS model for predicting maximum free surface oscillation in swaying rectangular storage tanks, Computers \& Mathematics with Applications, 76 (2018) 2496-2513.

[65] P. Bo, M. Bartoň, On initialization of milling paths for 5-axis flank CNC machining of free-form surfaces with general milling tools, Computer Aided Geometric Design, 71 (2019) 30-42.

[66] A. Calleja, P. Bo, H. González, M. Bartoň, L.N. López de Lacalle, Highly accurate 5-axis flank CNC machining with conical tools, The International Journal of Advanced Manufacturing Technology, 97 (2018) $1605-1615$.

[67] A. Hashemian, S.F. Hosseini, An integrated fitting and fairing approach for object reconstruction using smooth NURBS curves and surfaces, Computers \& Mathematics with Applications, 76 (2018) 1555-1575.

[68] A. Hashemian, B.M. Imani, A New Quality Appearance Evaluation Technique for Automotive Bodies Including Effect of Flexible Parts Tolerances, Mechanics Based Design of Structures and Machines, 46 (2018) 157-167.

[69] A. Hashemian, B.M. Imani, Surface Fairness: A Quality Metric for Aesthetic Assessment of Compliant Automotive Bodies, Journal of Engineering Design, 29 (2018) 41-64.

[70] J. Hinz, M. Möller, C. Vuik, Elliptic grid generation techniques in the framework of isogeometric analysis applications, Computer Aided Geometric Design, 65 (2018) 48-75. 
[71] H. Casquero, L. Liu, Y. Zhang, A. Reali, J. Kiendl, H. Gomez, Arbitrary-degree T-splines for isogeometric analysis of fully nonlinear Kirchhoff-Love shells, Computer-Aided Design, 82 (2017) 140153.

[72] L. Piegl, W. Tiller, The NURBS Book, 2nd ed., Springer-Verlag, New York, NY, 1997.

[73] A. Ebrahimi-Fizik, E. Lakzian, A. Hashemian, Entropy generation analysis of wet-steam flow with variation of expansion rate using NURBS-based meshing technique, International Journal of Heat and Mass Transfer, 139 (2019) 399-411.

[74] A.V. Vuong, C. Heinrich, B. Simeon, ISOGAT: A 2D tutorial MATLAB code for Isogeometric Analysis, Computer Aided Geometric Design, 27 (2010) 644-655.

[75] H.K. Versteeg, W. Malalasekera, An Introduction to Computational Fluid Dynamics: The Finite Volume Method, 2nd ed., Pearson Education Ltd., Harlow, UK, 2007.

[76] D.F. Griffiths, J.W. Dold, D.J. Silvester, Essential Partial Differential Equations: Analytical and Computational Aspects, Springer International Publishing, New York, NY, 2015.

[77] F. Bakhtar, Z.A. Mamat, O.C. Jadayel, On the performance of a cascade of improved turbine nozzle blades in nucleating steam. Part 2: Wake traverses, Proceedings of the Institution of Mechanical Engineers, Part C: Journal of Mechanical Engineering Science, 223 (2009) 1915-1929.

[78] K. Nordanger, R. Holdahl, T. Kvamsdal, A.M. Kvarving, A. Rasheed, Simulation of airflow past a 2D NACA0015 airfoil using an isogeometric incompressible Navier-Stokes solver with the Spalart-Allmaras turbulence model, Computer Methods in Applied Mechanics and Engineering, 290 (2015) 183-208.

[79] J.A. Cottrell, T.J.R. Hughes, Y. Bazilevs, Isogeometric Analysis: Toward Integration of CAD and FEA, John Wiley and Sons, Singapore, 2009.

[80] C.H. Kim, J.H. Park, D.I. Kim, J.H. Baek, Numerical analysis of non-equilibrium steam condensing flows in various Laval nozzles and cascades, Engineering Applications of Computational Fluid Mechanics, 11 (2017) 172-183.

[81] M.-C. Hsu, I. Akkerman, Y. Bazilevs, High-performance computing of wind turbine aerodynamics using isogeometric analysis, Computers \& Fluids, 49 (2011) 93-100.

[82] F. Bakhtar, R.J.K. Henson, H. Mashmoushy, On the Performance of a Cascade of Turbine Rotor Tip Section Blading in Wet Steam. Part 5: Theoretical Treatment, Proceedings of the Institution of Mechanical Engineers, Part C: Journal of Mechanical Engineering Science, 220 (2006) 457-472.

[83] M.J. Moore, P.T. Walters, R.I. Crane, B.J. Davidson, Predicting The Fog-Drop Size In Wet-Steam Turbines, in: 4th Wet Steam Conference, Coventry, UK, (1973). 